\title{
An approximation approach to a trade-off among efficiency, efficacy, and Balance for relief pre-positioning in disaster management
}

Rezaei-Malek M., Tavakkoli-Moghaddam R., Cheikhrouhou N., Taheri-Moghaddam

\begin{abstract}
This work develops a multi-objective, two-stage stochastic, non-linear, and mixed-integer mathematical model for relief pre-positioning in disaster management. Improved imbalance and efficacy measures incorporated into the model based on a new utility level of the delivered relief commodities. Moreover, this model considers the usage possibility of a set of alternative routes for each of applied transportation modes and consequently improves the network reliability. An integrated separable programming-augmented $\varepsilon$ constraint approach is proposed to address the problem. The best Pareto-optimal solution is selected by PROMETHEE-II. Finally, the theoretical improvements of the presented approach are validated by experiments and a real case study.
\end{abstract}

Keywords: Disaster management; Relief pre-positioning; Utility level; Efficacy; Balance; Multi-objective optimization.

\section{Introduction}

In 2013, natural disasters killed 21,610 persons, made 96.5 million victims, and caused around US\$ 156.7 billion worth of damages (Guha-Sapir et al., 2014). Disaster management approaches can help to mitigate the impacts on humans' lives on the basis of the development of adapted Humanitarian Relief Logistics (HRL) networks (Galindo and Batta, 2013). The Fritz Institute defines HRL as "the process of planning, implementing and controlling the effective, cost-efficient flow and storage of goods and materials as well as related information, from the point of origin to the point of consumption for the purpose of meeting the end beneficiary's requirements" (Thomas and Mizushima, 2005). There are some important tasks that fall under the broad umbrella of the HRL operations, e.g. preparedness, planning, procurement, transport, warehousing, tracking and tracing, and customs clearance (Thomas and Mizushima, 2005).

The HRL research is dedicated to the three planning stages in the disaster lifecycle: preparedness/pre-disaster phase, response and recovery phases (Özdamar and Ertem, 2015). Özdamar and Ertem (2015) presented a comprehensive literature review on the models, solutions and enabling technologies at the response and recovery phases.

At the preparedness phase, which is the domain of this work, various strategic decisions and procedures are devised before a disaster really occurs. For instance, the 
decisions about the number and locations of main Distribution Centers (DCs) to be opened and the amount of Relief Commodities (RCs) (e.g., non-perishable foods, medical supplies, clothes, blankets and tents) to be pre-positioned (Ahmadi et al., 2015). In fact, RCs can be secured or even purchased and pre-positioned at the preparedness phase (Altay and Green III, 2006). Humanitarian organizations can improve the agility of the HRL operations and appropriately respond to emergency situations if they establish a pre-determined network in which the location and required quantity of RCs are decided in advance that a disaster occurs (Rawls and Turquist, 2010). The aforementioned problem is known as Location with Relief Distribution and Stock Pre-positioning (LRDSP) problem in the HRL literature (Caunhye et al., 2012).

The initial studies that dealt with the relief pre-positioning problem in disaster management were about oil spills (e.g., see Psaraftis et al. (1986), Wilhelm and Srinivasa (1996), and Iakovou et al. (1997)). Later, Akkihal (2004) addressed the relief prepositioning problem in humanitarian and disaster relief management through a facility location approach. His work concentrated on determining locations that optimize worldwide humanitarian operations. He developed a methodology which applies integer programming to minimize the distance from warehouses to people who are likely to require humanitarian aid. After Akkihal (2004), many authors dealt with the relief prepositioning problem. In this regard, Caunhye et al. (2012) reviewed the literature of emergency optimization models. They explored some of the papers that considered the LRDSP problem. In addition, Hoyos et al. (2015) surveyed the published papers in the LRDSP category dealing with inherent uncertainty of the disaster area by stochastic components. These optimization models concurrently determine the optimal decisions on facility location, relief distribution and stock pre-positioning.

Jia et al. (2007) proposed a maximal-covering location model for the LRDSP problem. As extension of this work, Balcik and Beaman (2008) determined not only the number and locations of DCs, but also the quantity of pre-positioned RCs at each DC. The MILP model attempted to maximize the total expected demand covered by the established DCs (as a measure of efficacy), while captured budgetary, capacity and response time restrictions. They addressed the uncertainty of the demand of DPs by a scenario-based approach.

Rawls and Turquist (2010) coped with the LRDSP problem by a location-allocation approach and proposed a two-stage stochastic, mixed-integer program to minimize the expected costs over all scenarios while capturing facility capacity restrictions. They considered the uncertainties about undamaged amounts of the stocked RCs and capacity of the transportation network by a scenario-based approach. The costs were related to the selection of locations and sizes of facilities, RC acquisition and stocking, shipment of RCs to DPs (as measures of efficiency), unsatisfied demand penalties and holding costs for unused RCs (as measures of efficacy). Mete and Zabinsky (2010) developed a twostage stochastic program for the storage and distribution problem of Medical Supplies (MSs) in disaster management. They incorporated a restriction into the model to assure that the amount of unmet demand at each hospital cannot exceed a predefined threshold. The objective function of the model was to minimize the total cost of operating 
warehouses (as a measure of efficiency), and the expected value of total transportation duration and the penalty of unfulfilled demand (as measures of efficacy) with respect to predetermined disaster scenarios. They handle the uncertainty of required time to transport MSs to the hospitals using a scenario-based approach.

Regarding the consideration of the different kinds of natural disasters (i.e., flood and earthquake) and their relief requirements, Duran et al. (2011) developed a model to evaluate the effect of pre-positioning RCs on the CARE organization's average relief-aid emergency response time. The proposed MILP model captured the constraints about facility capacity, supply, demand and number of warehouses to be opened, and took account the uncertainty of number of people affected and their demand under different demand instances regarding the different disaster types. They considered the average of the weighted response times (as a measure of efficacy) to be minimized over the demand instances, while the former studies attempted to design a more cost-efficient network.

Rawls and Turquist (2011) defined a reliable set of scenarios and added some constraints to ensure a certain level of service quality in the selected scenarios as parts of the reliability set. Rawls and Turquist (2012) extended their previous papers to present a dynamic allocation model for optimizing the preparedness planning. The considered uncertainties were related to demands and their locations.

Döyen et al. (2012) presented a two-echelon, two-stage stochastic programming model for the LRDSP problem, where decisions were made to determine the location of regional and local rescue centers, the amount of RCs to be kept at the pre-disaster regional rescue centers, and the amount of RCs flows at each echelon.

A robust two-stage stochastic approach was developed by Bozorgi-Amiri et al. (2013) for a multi-objective disaster relief logistics network to determine the location of DCs and RCs allocation while capturing the facility capacity restrictions. In addition, minimization of the sum of maximum shortage at DPs (as a measure of efficacy) was considered as the second objective function of their model. Barzinpour and Esmaeili (2014) contributed to the literature by using the Proactive damage estimation result of Risk Assessment tool for Diagnosis of Urban Areas against Seismic Disaster software as an input to assign the affected people to local facilities that should be opened.

Rezaei-Malek and Tavakkoli-Moghaddam (2014) developed a robust bi-objective mixed-integer mathematical model for HRL network planning. They simultaneously considered minimization of the average weighted response time (as a measure of efficacy) and the total cost (as a measure of efficacy) as the objective functions. In the same year, Garrido et al. (2014) assumed there are different RCs that need different types of transportation modes to be delivered to DPs. For instance, medications may need reefer vehicles while fresh water need tankers. Therefore, they considered different vehicles' classes so that each RC can be transported by capable vehicle. Ahmadi et al. (2015) proposed a two-stage stochastic, multi-depot, location-routing model considering random travel time, multiple uses of vehicles and standard relief time. Their model determines the locations of local depots and routing for last mile distribution after an earthquake. Rodriguez-Espindola and Gaytan (2015) contributed to the LRDSP literature through a concurrent determination of the location of emergency shelters and DCs along 
with an allocation of required RCs to DCs. They presented a bi-objective mathematical model. The first objective minimized the sum of the acquisition costs, shipping costs and facility preparation costs (as a measure of efficiency), and the second one minimized the total priority-weighted distance travelled by goods and people (as an efficacy measure). They considered a multi-mode transportation system for the HRL network; however, they did not tack account the usage possibility of alternative routes for each of the employed transportation mode. This feature increases the reliability of the transportation system when some routes may be completely or partially destroyed after a disaster strikes. Tofighi et al. (2015) developed a two-stage scenario-based possibilistic-stochastic programming model to design a HRL network. In the first stage, the locations of multiple Central Warehouses (CWs) and local DCs were specified along with the quantity of pre-positioned RCs. In the next stage, a RCs distribution plan was presented for the different scenarios. In addition, they developed a tailored DE algorithm to achieve sufficient and feasible solutions in a reasonable CPU time. The model minimized the total operating costs of selected CWs and Local DCs and their inventory costs (as a measure of efficiency), the total quantity-weighted distribution times, maximum priority-weighted travel time between each pair of CW/Local DC and DP, and the total cost of unused inventories and priority-weighted shortage cost (as measures of efficacy). Rezaei-Malek et al. (2016) developed a multi-objective programming model to design a disaster relief logistics network. They considered the lifetime of perishable products (e.g., medical commodities and packed milk) by adjusting certain time windows.

Distribution of RCs from supply points to Demand Points (DPs) is generally planned through pre-determined routes and transportation modes (e.g., considered at Rawls and Turquist (2010) and Rezaei-Malek and Tavakkoli-Moghaddam (2014)). The required time to transport RCs to DPs by different transportation modes often is estimated regarding the disaster scenarios, because a specific disaster, such as an earthquake, may devastate the routes partially or completely and affect transportation times. On the other side, there is an uncertainty on available transportation modes and vehicles when a disaster strikes. Indeed, the usage possibility of different alternative routes for transporting RCs, can increase the reliability of a HRL network (e.g. considered at Hamedi et al. (2012)). In other words, when a predetermined relief route is completely destroyed, the existence of some alternative available routes increases the responsiveness of the network. Furthermore, the consideration of different transportation modes improves the reliability of the system (e.g. considered at Ruan et al. (2014)). For example, when air rescue is impossible because of a bad weather condition, land transportation can be a great alternative if the network is enabled in advance. Therefore, the usage possibility of alternative routes for each applied transportation mode definitely increases the reliability and responsiveness of the network. This such an integrated feature remains unattached in the current literature of the LRDSP problem (see Table 1).

Gutjahr and Nolz (2016) reviewed the recent literature on the application of multicriteria optimization to disaster management. Efficiency, efficacy, equity, and happiness (or in its negative form, distress) are four significant criteria that have to be considered in HRL network design (Gutjahr and Nolz, 2016). Efficiency means cost-efficiency and is 
generally measured by logistics cost. The logistics cost includes (i) fixed costs for the procurement of equipment (e.g., vehicles), (ii) supply-side traveling costs related to the transportation of RCs and people by aid-delivering parties, (iii) facility-related costs such as facility opening costs and inventory holding costs, and (iv) costs related to the human resources such as wages of employed personnel (Gutjahr and Nolz, 2016). Efficacy of a HRL can be expressed by the different measures or a combination of them such as response time, travel distance, coverage, reliability, and security (Gutjahr and Nolz, 2016). Noteworthy, in a disaster area, the efficacy of a network is more important than its efficiency because saving human lives has a higher priority than monetary issues (Balcik and Beaman, 2008). On the other side, it is a natural requirement that the distribution of RCs (of whatever form) should be fair, which means that it should be no privilege for certain groups of individuals. Fair distribution is usually captured by the concept of equity (Gutjahr and Nolz, 2016). Indeed, the equity concept is often explored in allocation problems. In these problems, resources or commodities should be assigned to a set of entities (e.g., organizations, people or groups of individuals at different DPs). The consideration of this concept in an allocation problem means servicing a set of entities in a "fair" manner (Karsu and Morton, 2015). There are two equity-related concepts called equitability and balance. The equitability concept means servicing a set of indistinguishable entities while the balance concept means servicing a set of entities while they are different from each other regarding their needs, claims, and preferences. In the latter concept, an ideal solution may give each entity a different proportion of the total assignment. The balance concept must be considered in various real life allocation problems (Karsu and Morton, 2015). For an instance, in disaster areas the different DPs have different demand amount and priority because of different criteria (e.g., population and severity of disaster) (Gralla et al., 2014; Nagurney et al., 2015). Hence, balance should be satisfied among DPs in the distribution of RCs for preventing a possible social disaster (Rezaei-Malek and Tavakkoli-Moghaddam, 2014). In other word, taking this criterion into account is necessary in the distribution plan of a HRL network and people expect to observe respecting balance concern among DPs in the distribution plan (Altay and Green III, 2006). In this regard, Rezaei-Malek and Tavakkoli-Moghaddam (2014) proposed a set of constraints to ensure that the difference of priority-weighted shortage between each two DPs does not exceed a threshold defined by experts. On the other side, since the HRL networks often face shortage to satisfy all the demands, it is reasonable to use the proportion of demand satisfied as a measure of the balance concept (Karsu and Morton, 2015). In this respect, Davis et al. (2013) presented a new model satisfying a minimum fraction of demand at all of the DPs. They introduced a new constraint for distinguishing among the DPs with regard to their respective demands. In a similar way, Vitoriano et al. (2011) considered the same measure and minimized its deviation from a predetermined target for all of the DPs. Tzeng et al. (2007) attempted to maximize the minimum level of this measure for all of the RCs. Huang et al. (2012) introduced and then maximized a convex disutility function $f(w)$, where $w$ was the fraction of unsatisfied demand, to reflect the nonlinear change rate of this disutility. 
The distress concept refers to psychological and social costs which are addressed only in a minority of the surveyed papers. Indeed, in addition to judging the efficiency of a HRL in monetary terms, it should be assessed regarding the extent by which it decreases deprivation, hardship, affliction, pain, negative emotions, and social disturbances of the affected people (Karsu and Morton, 2015). In this respect, Holguín-Veras et al. (2013) introduced the concept of deprivation costs to quantify the suffering of affected people lacking vital RCs. They expressed that the deprivation cost of a specific RC is seen as a non-decreasing convex function of the time that has passed since the demand for this RC has been fulfilled (Holguín-Veras et al., 2013). Indeed, the value of RCs is decreasing while delivery time is postponing because the number of victims and people suffering are increasing (Edrissi et al., 2013).

This paper inspired by Huang et al. (2012), Holguín-Veras et al. (2013) and RezaeiMalek and Tavakkoli-Moghaddam (2014) develops new efficacy and imbalance measures. The new efficacy measure is the expected time-, priority-, and demandweighted utility levels of the delivered RCs (MR utility levels) at all the DPs in the HRL network. The new imbalance measure (inspired by Rezaei-Malek and TavakkoliMoghaddam (2014)) is the expected maximum difference of the MR utility levels among the DPs. We calculated the MR utility level at each specific DP regarding the delivery time, priorities of RCs and DP, and fraction of satisfied demand. The utility of delivery time is considered as a decreasing convex function of the time (inspired by Holguín-Veras et al. (2013) as a deprivation cost), and the utility of fraction of satisfied demand is formulated as an increasing convex function of the fraction of fulfilled demand (inspired by Huang et al. (2012)). In addition, this new utility level of the delivered RCs (i.e., the MR utility level) for each specific DP considers the priorities of different RCs and DP under each disaster scenario.

Table 1 summarizes the LRDSP literature and depicts the features of the different developed models for the LRDSP problem. Briefly, the contributions of this paper are as follows:

- Taking into account the usage possibility of a set of alternative routes for each applied transportation mode in HRL networks.

- Introducing a new time-, priority-, and demand-weighted utility level of the delivered RCs, called MR utility level.

- Proposing new efficacy and imbalance measures based on the MR utility level.

- Presenting an integrated separable programming-augmented $\varepsilon$-constraint approach to deal with the presented multi-objective nonlinear model.

Table 1

LRDSP literature and its features

\begin{tabular}{|c|c|c|c|c|c|c|c|c|}
\hline Author(s) & Model & MOP & Uncertainty & Solution & $\begin{array}{l}\text { Multi- } \\
\text { mode }\end{array}$ & $\begin{array}{l}\text { Multi- } \\
\text { route }\end{array}$ & $\begin{array}{l}\text { Multi- } \\
\text { item }\end{array}$ & $\begin{array}{l}\text { Case } \\
\text { study }\end{array}$ \\
\hline $\begin{array}{l}\text { Psaraftis et al. } \\
\text { (1986) }\end{array}$ & MILP & & $S$ & $\mathrm{H}$ & & & $*$ & * \\
\hline $\begin{array}{l}\text { Wilhelm and } \\
\text { Srinivasa } \\
\text { (1996) }\end{array}$ & MILP & & $S$ & $\mathrm{H}$ & & & * & $*$ \\
\hline
\end{tabular}




\begin{tabular}{|c|c|c|c|c|c|c|c|c|}
\hline $\begin{array}{l}\text { Iakovou et al. } \\
\text { (1996) }\end{array}$ & LIP & & & E & & & * & * \\
\hline McCall (2006) & MILP & & & $\mathrm{E}$ & & & $*$ & * \\
\hline $\begin{array}{l}\text { Chang et al. } \\
\text { (2007) }\end{array}$ & MILP & & S & $\mathrm{H}$ & & & * & * \\
\hline $\begin{array}{l}\text { Balcik and } \\
\text { Beamon } \\
(2008)\end{array}$ & MILP & & $S$ & $\mathrm{E}$ & & & $*$ & * \\
\hline $\begin{array}{l}\text { Mete and } \\
\text { Zabinsky } \\
(2010)\end{array}$ & MILP & & S & $\mathrm{E}$ & & $*$ & $*$ & $*$ \\
\hline $\begin{array}{c}\text { Rawls and } \\
\text { Turnquist } \\
\text { (2010) }\end{array}$ & MILP & & $S$ & $\mathrm{H}$ & & & $*$ & $*$ \\
\hline $\begin{array}{l}\text { Huang and } \\
\text { Fan (2011) }\end{array}$ & LIP & & S & E & & & $*$ & * \\
\hline Li et al. (2011) & MILP & & S & $\mathrm{H}$ & & $*$ & & $*$ \\
\hline $\begin{array}{l}\text { Rawls and } \\
\text { Turnquist } \\
\text { (2011) }\end{array}$ & MILP & & $S$ & $\mathrm{E}$ & & & $*$ & $*$ \\
\hline $\begin{array}{l}\text { Duran et al. } \\
\text { (2011) }\end{array}$ & MILP & & $S$ & $\mathrm{E}$ & & & * & * \\
\hline $\begin{array}{l}\text { Tofighi et al. } \\
\text { (2011) }\end{array}$ & MILP & & $\mathrm{F}, \mathrm{S}$ & $\mathrm{E}$ & & & $*$ & $*$ \\
\hline $\begin{array}{l}\text { Rawls and } \\
\text { Turnquist } \\
(2012)\end{array}$ & DMILP & & $S$ & $\mathrm{E}$ & & & $*$ & * \\
\hline $\begin{array}{l}\text { Bozorgi-Amiri } \\
\text { et al. (2012) }\end{array}$ & MILP & & $\mathrm{S}, \mathrm{R}$ & MH & & & * & \\
\hline $\begin{array}{l}\text { Döyen et al. } \\
\text { (2012) }\end{array}$ & MILP & & S & $\mathrm{H}$ & & & $*$ & \\
\hline $\begin{array}{l}\text { Bozorgi-Amiri } \\
\text { et al. (2013) }\end{array}$ & MILP & $\mathrm{EF}, \mathrm{EC}$ & $\mathrm{S}, \mathrm{R}$ & E & & & $*$ & * \\
\hline $\begin{array}{l}\text { Garrido et al. } \\
\quad(2014)\end{array}$ & DMILP & & $S$ & $\mathrm{H}$ & $*$ & & $*$ & \\
\hline $\begin{array}{c}\text { Rezaei-Malek } \\
\text { and } \\
\text { Tavakkoli- } \\
\text { Moghaddam } \\
\text { (2014) }\end{array}$ & MILP & $\mathrm{EF}, \mathrm{EC}$ & $\mathrm{S}, \mathrm{R}$ & $\mathrm{E}$ & & & $*$ & * \\
\hline $\begin{array}{c}\text { Barzinpour } \\
\text { and Esmaeili } \\
(2014)\end{array}$ & MILP & $\mathrm{EF}, \mathrm{EC}$ & & $\mathrm{E}$ & & & $*$ & $*$ \\
\hline $\begin{array}{l}\text { Lee et al. } \\
(2014)\end{array}$ & MILP & & S & E & & & * & * \\
\hline $\begin{array}{l}\text { Ahmadi et al. } \\
\text { (2015) }\end{array}$ & MILP & & S & $\mathrm{H}$ & & & * & * \\
\hline $\begin{array}{l}\text { Rodriguez- } \\
\text { Espindola and } \\
\text { Gaytan (2015) }\end{array}$ & MILP & $\mathrm{EF}, \mathrm{EC}$ & & $\mathrm{E}$ & $*$ & & $*$ & * \\
\hline $\begin{array}{l}\text { Tofighi et al. } \\
\text { (2015) }\end{array}$ & MILP & $\mathrm{EF}, \mathrm{EC}$ & $\mathrm{S}, \mathrm{P}$ & $\mathrm{H}$ & & & $*$ & * \\
\hline $\begin{array}{l}\text { Rezaei-Malek } \\
\text { et al. (2016) }\end{array}$ & MILP & $\mathrm{EF}, \mathrm{EC}$ & $\mathrm{S}, \mathrm{R}$ & E & & & $*$ & * \\
\hline Present work & MINLP & EF,EC,IB & $\mathrm{S}$ & $\mathrm{A}$ & $*$ & $*$ & $*$ & $*$ \\
\hline
\end{tabular}

MILP: Mixed-Integer Linear Programming, LIP: Linear Integer Programming, DMILP: Dynamic Mixed-Integer Programming, S: Stochastic, F: Fuzzy, R: Robust, P: Possibilistic, H: Heuristics, E: Exact with commercial software, MH: Meta-heuristics, EF: Efficiency, EC: Efficacy, EQ: Equitability. IB: Imbalance, A: Approximate. 
The rest of this paper is organized as follows. In Section 2, the problem and the variables are modelled and a multi-objective mathematical model is developed. Section 3 delineates the solution approach proposed. The computational experiments are provided in Section 4. In Section 5, a real case is described with the aim to prove the applicability of the approach. Finally, Section 6 draws the conclusions and future research directions.

\section{Problem description and mathematical formulation}

We consider a HRL network consisting of warehouses and DPs. There is a set of candidate warehouses to be selected for satisfying the demand of DPs. The set of candidate warehouses is denoted by $I$ and indexed by $i \in I$. Let $y_{i}$ be a binary decision variable equals to 1 if warehouse $i$ is selected, and 0 otherwise. Opening warehouse $i$ incurs a fixed $\operatorname{cost} F_{i}$ that includes costs of opening, personnel, maintenance, etc.

The locations of DPs are known. The set of DPs is denoted by $J$ and indexed by $j \in J$. The problem is to determine the location, allocation and distribution plan of each RC in the network. The set of RCs is denoted by $K$ and indexed by $k \in K$. Selected warehouses should store RCs in appropriate quantities with regard to their capacity limitation. The capacity of warehouse $i$ for RC $k$ is known through $\gamma_{i k}$. Let $q_{i k}$ be the amount of RC $k$ that should be stored at warehouse $i$.

There are different connection routes (i.e., overland routes and the shortest airway and waterway) between each pair of warehouse and DP. Let $A_{i j v}$ be a known set of alternative routes between warehouse $i$ and DP $j$ that can be passed by vehicle $v$. Noteworthy, the demand of each DP can be satisfied by the different warehouses (i.e., multi-allocation) through using different modes of transportation (i.e., aviation, ship, and land transport). The densities of RCs are negligible, so the volume capacity of vehicles (e.g., aircrafts, ships, trucks, and trains) are considered as a restriction for loading RCs. So, let $v_{k}$ be the volume of RC $k$, and $V_{v}$ denotes the total capacity of each vehicle $v$. The average transportation cost of a unit RC by vehicle $v$ denotes by $T C^{v}$. Indeed, this paper considers the related operational costs to the response phase (i.e., the transportation cost) and takes account the costs related to the preparedness phase including opening the required warehouses, pre-positioning the needed RCs.

There is a limitation on the available amount of RC $k$ for pre-positioning at the warehouses $\left(\xi_{k}\right)$. The unit acquisition cost of RC $k$ is denoted by $A C_{k}$, and for preventing a RCs congestion in the warehouses after a disaster occurrence, an additional unit holding cost of RC $k$ (i.e., $h_{k}$ ) is considered for the unused amount of RC $k$ in warehouse $i$ in scenario $s$ (i.e., $z_{i k s}$ ). Uncertainty is modeled through the determination of a set $S$ of discrete scenarios indexed by $s \in S$, each with a probability of occurrence $p_{s}$.

The demand of DP $j$ for RC $k$ in scenario $s$ is $d_{j k s .}$ Let $x_{i j k}^{v r s}$ be the quantity of RC $k$ should be sent to DP $j$ from warehouse $i$ by vehicle $v$ through route $r$ in scenario $s$. If the demand for a particular RC $k$ cannot be met, $w_{j k s}$ is considered as the shortage of RC $k$ in DP $j$ in scenario $s$. The parameter $\tau_{j s}$ implies the priority of DP $j$ in scenario $s$, and the parameter $\xi_{k}$ denotes the importance weight of RC $k$ in a disaster. These parameters will be determined according to the experts' opinion of an HRL organization about the severity 
and situation of disaster (Sheu, 2010). The delivery time of the required RCs at DPs, impacts on the utility level of the delivered RCs. This utility reduces to the lower levels when more time passes, because more people lose their lives (Edrissi et al., 2013). Let $t_{i j}^{v r s}$ be the time taken for the relief team of warehouse $i$ to reach DP $j$ by vehicle $v$ through route $r$ in scenario $s$.

Assume that variable $t_{i j}^{\text {vrs }}$ represents the transportation time between warehouse $i$ and DP $j$ reflecting the road and traffic conditions related to the impact of disaster scenario $s$ on route $r$. According to the above discussion, let $\Xi\left(t_{i j}^{v r s}\right)$ be a utility level function of the delivery time. A typical shape for this function is depicted in Fig. 1. As shown in the figure, any increase in $t_{i j}^{v r s}$ decreases the utility level of the delivered RCs, and this effect also becomes more dramatic after 12 hours (i.e., standard relief time) (Ahmadi et al., 2015). In other words, the shape addresses this fact that the initial hours after a disaster are very important for serving the affected people. Moreover, $\Xi(t)$ converges to 0 after $72 \mathrm{~h}$ because the required RCs become useless for saving the injured people after $72 \mathrm{~h}$ in the initial response phase (Edrissi et al., 2013).

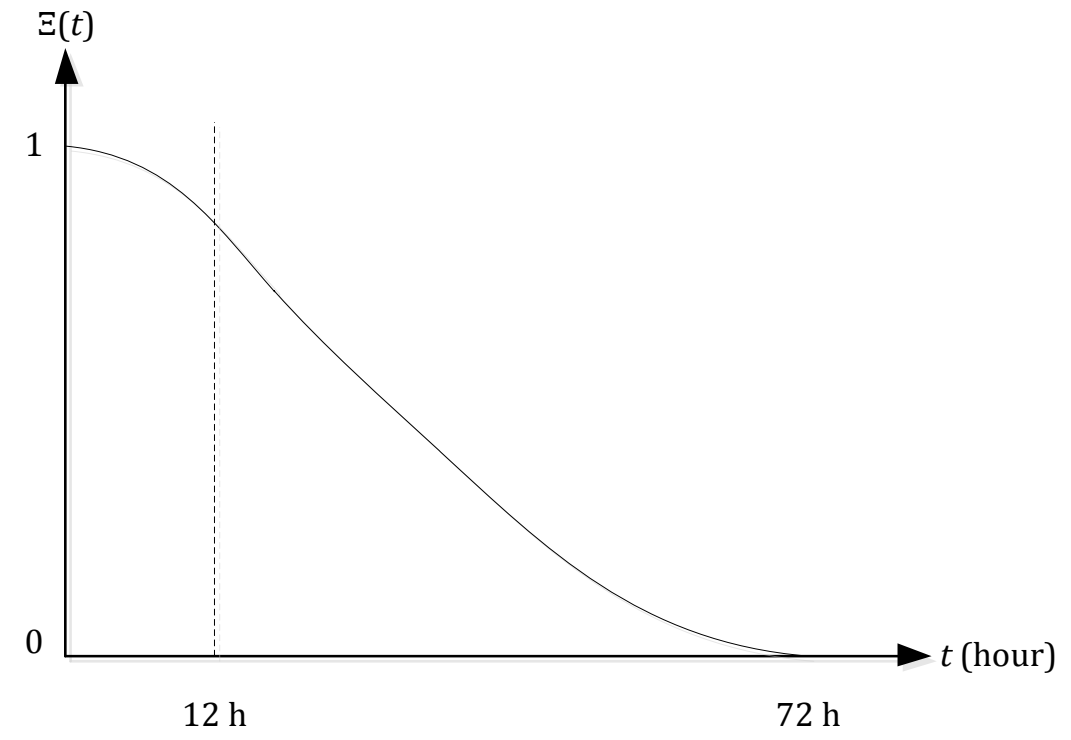

Fig. 1. Shape of the utility level for the delivery time of RCs

Inspired by the work of Huang et al. (2012), this paper considers a utility function of the fraction of satisfied demand, $f(o)$, where $o$ is the fraction of the satisfied demand. This function reflects that the increase of the utility level for the delivered RCs is greater when the fraction of the satisfied demand is closer to 1 (see Fig. 2). 


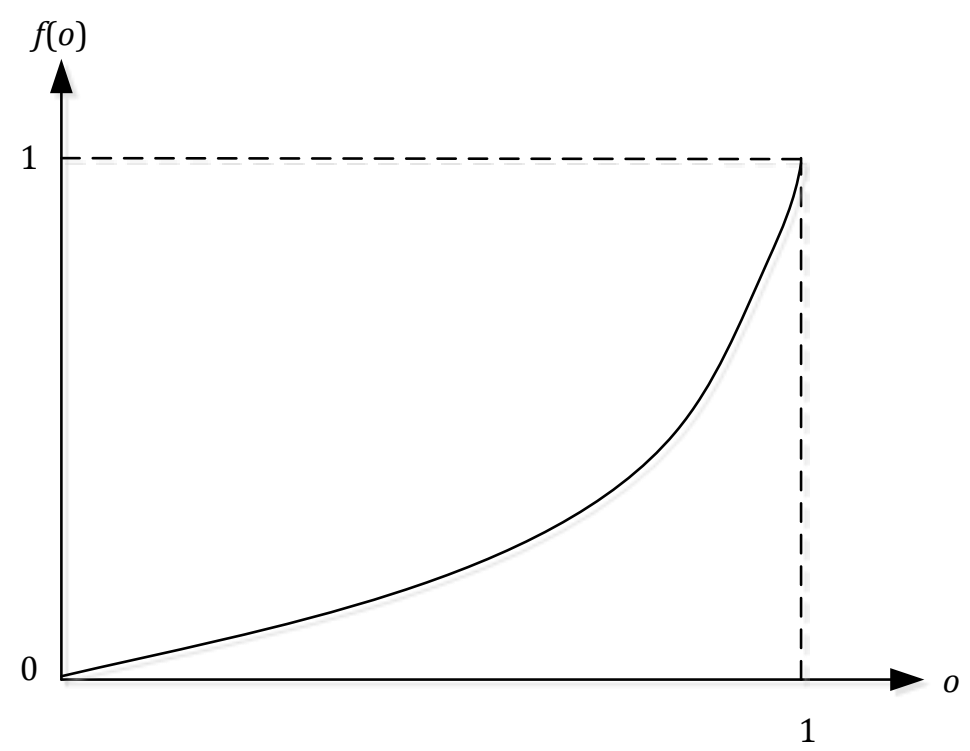

Fig. 2. Shape of the utility function for the fraction of the satisfied demand

Warehouses may be affected by the disaster so that the complete or partial amount of stocked RCs may be destroyed. Hence, let $\rho_{i k s}$ be the percentage of stocked materials of $\mathrm{RC} k$ at warehouse $i$ that remains usable in scenario $s\left(0 \leq \rho_{i k s} \leq 1\right)$.

$\psi_{j k s}$ is the tolerable proportion of shortage in DP $j$ for RC $k$ in scenario $s$ that ensures the minimum delivery of RCs to the DPs. We seek to maximize the efficiency and efficacy of the HRL network while minimizing the expected maximum difference of the MR utility levels among DPs over all the scenarios to prevent a probable social disaster that may happen because of not satisfying balance in distribution of RCs among the DPs.

In our problem setting, a scenario-based modeling is applied to include a number of discrete scenarios, which account for abovementioned uncertainties following different scenarios. Each scenario is associated with a given likelihood $\left(p_{s}\right)$. Accordingly, a multiobjective, two-stage stochastic, non-linear, and mixed-integer program with recourse is developed to formulate the HRL problem.

Noteworthy, the two-stage stochastic programming is one of the most widely employed approaches to cope with two-stage decision problems. In a two-stage model, an initial decision is made in the stage one before being informed what scenario will be happened and what values the scenario-dependent parameters will take at the stage two. Therefore, a recourse action is taken in the stage two to compensate for the decision made in the stage one (Falasca and Zobel, 2011).

In the decision problem under consideration, determining the warehouses that should be opened and the amount of RCs that should be prepositioned at the pre-disaster phase (stage 1) constitute the first-stage decisions. These decisions must be made before the realization of any disaster scenario. Moreover, given the realized scenario at the postdisaster phase (stage 2), determining the quantity of RCs which must be sent from the opened warehouses to the DPs is the main second-stage/recourse decision. 
Below, we first define the notations, and then present the equivalent model of our multi-objective, two-stage stochastic, non-linear, and mixed-integer program. In the next section, we discuss how to convert this non-linear model into an approximate-linear one, and how to change the multi-objective model into an equivalent single-objective form. Notably, the presented counterpart single-objective and approximate-linear model can be directly solved by commercial optimization packages like GAMS.

\subsection{Indices}

$i \quad$ index of candidate warehouses $(i \in I)$,

$j \quad$ index of DPs $(j \in J)$,

$v \quad$ index of vehicles $(v \in V)$,

$r \quad$ index of routes $\left(r \in A_{i j v}\right)$,

$k \quad$ index of RCs $(k \in K)$,

$s \quad$ index of disaster scenarios $(s \in S)$.

\subsection{Deterministic parameters}

$F_{i} \quad$ fixed cost of opening warehouse $i$,

$A C_{k} \quad$ unit acquisition cost of RC $k$,

$T C^{v}$ average transportation cost of a unit RC by vehicle $v$ per time unit,

$\gamma_{i k} \quad$ capacity of warehouse $i$ for RC $k$ (unit),

$h_{k} \quad$ additional unit holding cost of RC $k$,

$\vartheta_{k} \quad$ available quantity of RC $k$ (unit),

$\xi_{k} \quad$ priority of RC $k$ when a disaster strikes; $\left(0 \leq \xi_{k} \leq 1 ; \sum_{k} \xi_{k}=1\right)$,

$A_{i j v} \quad$ a set of the known routes between warehouse $i$ and DP $j$ that can be used by vehicle $v)$,

$M \quad$ a sufficient big number.

\subsection{Uncertain parameters}

$p_{s} \quad$ probability of occurrence of disaster scenario $s ;\left(0 \leq p_{s} \leq 1 ; \sum_{s} p_{s}=1\right)$,

$t_{i j}^{\text {vrs }} \quad$ transportation time between warehouse $i$ and DP $j$ by vehicle $v$ through route $r$ in scenario $s$ (hour),

$\rho_{i k s} \quad$ proportion of stocked materials of RC $k$ at warehouse $i$ that remains usable in scenario $s ;\left(0 \leq \rho_{i k s} \leq 1\right)$,

$\tau_{j s} \quad$ priority of DP $j$ in scenario $s ;\left(0 \leq \tau_{j s} \leq 1 ; \sum_{j s} \tau_{j s}=1\right)$,

$d_{j k s} \quad$ demand of DP $j$ for RC $k$ in scenario $s$ (unit),

$\psi_{j k s} \quad$ tolerable proportion of shortage RC $k$ in DP $j$ in scenario $s ;\left(0 \leq \psi_{j k s} \leq 1\right)$,

\subsection{Scenario-independent (i.e., first-stage's) variables}

$q_{i k} \quad$ amount of RC $k$ pre-positioned at warehouse $i$,

$y_{i} \quad 1$; if the warehouse $i$ is opened, 0 ; otherwise. 


\subsection{Scenario-based (i.e., second-stage's) variables}

$x_{i j k}^{v r s} \quad$ quantity of RC $k$ sent to DP $j$ from warehouse $i$ by vehicle $v$ through route $r$ in scenario $s$,

Ziks amount of RC $k$ in warehouse $i$ that is not used in scenario $s$,

$w_{j k s} \quad$ shortage of RC $k$ in DP $j$ in scenario $s$,

$\varphi_{j s} \quad$ amount of the MR utility level at DP $j$ in scenario $s$,

$\delta_{s} \quad$ maximum difference of the MR utility levels among DPs in scenario $s$.

Fig. 3 depicts the two different stages and their related variables.

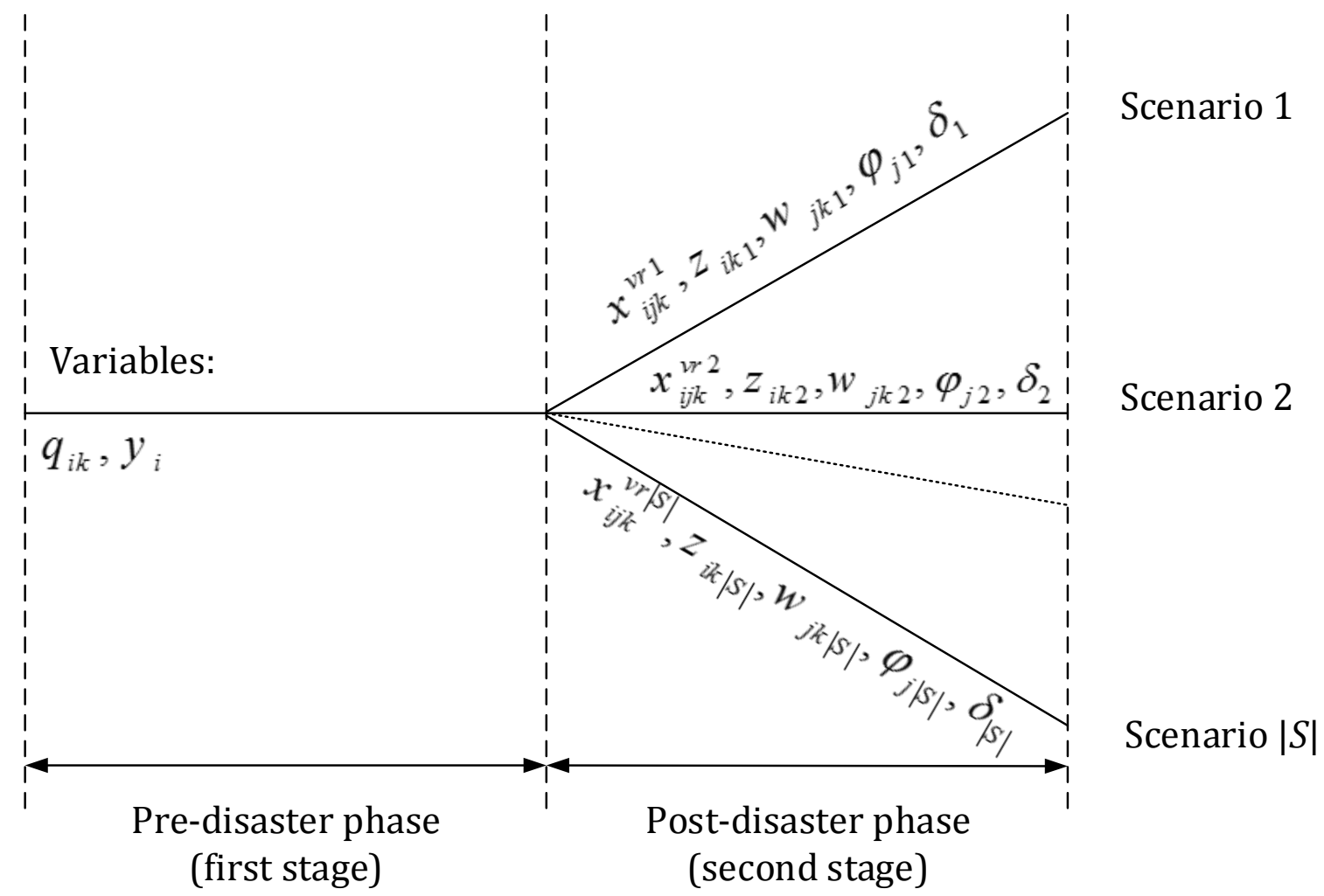

Fig. 3. Representation of two different stages and related variables.

\subsection{Proposed non-linear mathematical model (Model 1)}

Based on the above-mentioned definitions, we develop the following multi-objective, two-stage, mixed-integer nonlinear mathematical model. First, the set of the objective functions are described, and then the constraints sets are elaborated.

\subsubsection{Objective functions}

The formulation of the multiple objectives is presented as follows: 


$$
\operatorname{Min} \mathrm{OF} 1=\sum_{i \in I}\left(F_{i} y_{i}+\sum_{k \in K} A C_{k} q_{i k}\right)+\sum_{s \in S} p_{s} \sum_{i \in I} \sum_{k \in K}\left(\sum_{j \in J} \sum_{v \in V} \sum_{r \in A_{i j v}} T C^{k v} x_{i j k}^{v r s}+h_{k} z_{i k s}\right)
$$

$$
\begin{aligned}
& \text { Max OF2 }=\sum_{s \in S} \sum_{j \in J} p_{s} \varphi_{j s} \\
& \text { Min OF3 }=\sum_{s \in S} p_{s} \delta_{s}
\end{aligned}
$$

The objective function OF1 (1) considers the efficiency concern and minimizes the total cost at the pre-disaster phase consisting of the sum of the fixed cost of establishing warehouses (i.e., term 1), the fixed cost of pre-positioning RCs (i.e., term 2), and the total cost at the post-disaster phase including the transportation cost (i.e., term 3) and the additional holding penalty cost for unused RCs (i.e., term 4). The objective function OF2 (2) maximizes the expected time-, demand-, and priority-weighted utility level of the delivered RCs (i.e., the MR utility level) at the DPs over all the scenarios (i.e., the efficacy measure).The MR utility level at each DP, $\varphi_{j s}$, is calculated by Equation (10). The objective function (3) considers the imbalance measure and minimizes the expected maximum difference of the MR utility levels among DPs over all the scenarios. The maximum difference of the MR utility levels among DPs in scenario $s, \delta_{s}$, is obtained using the constraint (11).

\subsubsection{Constraints sets}

(I) RCs flow at the stage two

$$
\begin{aligned}
& x_{i j k}^{v r s} \leq M t_{i j}^{v r s} \quad \forall i \in I, j \in J, v \in V, r \in A_{i j v}, k \in K, s \in S \\
& \sum_{j \in J} \sum_{v \in V} \sum_{r \in A_{i j v}} x_{i j k}^{v r s}+z_{i k s}=\rho_{i k s} q_{i k} \quad \forall i \in I, k \in K, s \in S \\
& w_{j k s}+\sum_{i \in I} \sum_{v \in V} \sum_{r \in A_{i j v}} x_{i j k}^{v r s}=d_{j k s} \quad \forall j \in J, k \in K, s \in S \\
& w_{j k s} \leq \psi_{j k s} d_{j k s} \quad \forall j \in J, k \in K, s \in S
\end{aligned}
$$

Constraint (4) assures that the shipped RCs are delivered by vehicle $v$ through available routes (if a route is not available, its duration time is considered equal to zero). Equation (5) guarantees that the sum of the shipped RC $k$ and unused RC $k$ at warehouse $i$ is equal to the amount of undamaged stocked materials at warehouse $i$. Equation (6) ensures the sum of RCs sent to each DP and the amount of the unmet demand is equal to the demand of that DP. The model restricts the allowable amount of shortage for each DP by Constraint (7). 
(II) Open facilities and facility capacity

$$
\begin{aligned}
& \sum_{i=I} q_{i k} \leq \vartheta_{k} \quad \forall k \in K \\
& q_{i k} \leq y_{i} \gamma_{i k} \quad \forall i \in I, k \in K
\end{aligned}
$$

The restriction on the available amount of RC $k$ for pre-positioning in the selected warehouses is imposed by Constraint (8). Constraint (9) guarantees that if warehouse $i$ is opened, the corresponding amount of RC $k$ does not exceed the capacity of warehouse $i$.

(III) Imbalance among PDs

$$
\begin{aligned}
& \varphi_{j s}=\sum_{i \in I} \sum_{v \in V} \sum_{r \in R} \sum_{k \in K}\left(\left(\tau_{j s} \times \xi_{k}\right) \times f\left(\frac{x_{i j k}^{v r s}}{d_{j k s}}\right) \times \Xi\left(t_{i j}^{v r s}\right)\right) \\
& -\delta_{s} \leq \varphi_{p s}-\varphi_{q s} \leq \delta_{s} \quad \forall s, \forall p \neq q \in J
\end{aligned}
$$

Equation (10) is introduced for the first time in the literature. This equation calculates the priority-, demand-, and time-weighted utility level of the delivered RCs (i.e., the MR utility level) at DP $j$. Constraints (11) calculate the maximum difference of the MR utility levels among all the DPs following scenario $s$.

(IV) Non-negativity and binary constraints

$$
\begin{array}{ll}
x_{i j k}^{v r s} \geq 0 & \forall i \in I, j \in J, v \in V, r \in A_{i j v}, k \in K, s \in S \\
q_{i k} \geq 0 & \forall i \in I, k \in K \\
z_{i k s} \geq 0 & \forall i \in I, k \in K, s \in S \\
w_{j k s} \geq 0 \quad \forall j \in J, k \in K, s \in S \\
\delta_{s} \geq 0 \quad \forall s \in S \\
y_{i} \in\{0,1\} \quad \forall i \in I
\end{array}
$$

Equations (12) to (16) are non-negativity limitations, and Equation (17) indicates that opening a warehouse location is a binary decision.

\section{Methodology}

The term $f\left(x_{i j k}^{v r s} / d_{j k s}\right)$ introduces a non-linearity into the objective functions (2) and (3) of Model 1. Generally, non-linear programming models are more difficult to solve than the linear ones. Hence, we convert the presented non-linear model to a linear approximation form through separable programming. Employing the separable programming approach or its extended version depends upon a significant classification of non-linear models into convex and non-convex problems. In the case of non-convex programming, the original separable programming cannot guarantee a global optimal 
solution. However, in a convex programming model, any optimum found must be a global optimal solution (Williams, 2013). Obtaining a guaranteed global optimum to a nonconvex programming model requires a more complex algorithm than the original separable programming algorithm. A satisfactory, though often computationally expensive, way of dealing with the problem is to add binary variables and logical constraints into the original separable programming approach (see Williams (2013); p. 177-182).

After obtaining a linear approximation for Model 1 by applying the separable programming (see Section 3.1), the problem is converted to a multi-objective mixed integer linear mathematical model. Several methods have been developed in the logistics literature to tackle the multi-objective mathematical models such as the weighted sum, $\varepsilon$-constraint, Tchebycheff-based methods or the fuzzy programming (cf. for instance Rezaei-Malek and Tavakkoli-Moghaddam, 2012; Torabi et al., 2015; Salehi Sadghiani et al., , 2015; Sedighy et al., 2015; Zhalechian et al., 2016). We develop in this work an improved version of the $\varepsilon$-constraint method, namely augmented $\varepsilon$-constraint method, by which the multi-objective model is converted to a single objective counterpart. This method is an appropriate approach for our problem because it can handle non-convexity and assures the efficiency of the achieved solutions (i.e., Pareto optimal solutions). In order to select the most preferable solution among the Pareto optimal solutions, the PROMETHEE-II method is adopted because it is among the most efficient approaches when one faces a huge number of alternatives and criteria (Tzeng and Huang, 2011). The next sections elaborate the applied methods in the proposed methodology (see Fig. 4).

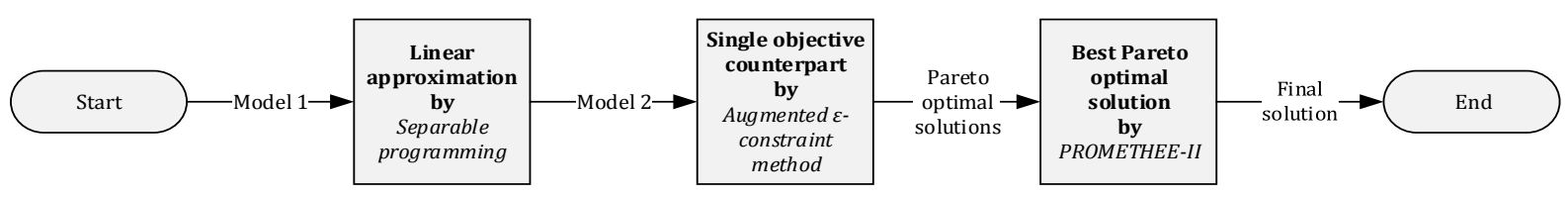

Fig. 4. Proposed methodology

\subsection{Separable programming}

In a separable programming approach, there is no difference between the required procedure to tackle a non-linear objective function, a constraint or both. Following, the manner to deal with a non-linear function is described.

First, the non-linear function should be separable. A separable function is a function that can be presented as the sum of functions of a single variable. Accordingly, the objective functions (2) and (3) are separable. In a mathematical programming model, the separable functions can be approximated to piecewise linear functions (Williams, 2013).

As it is obvious in the formulation, the only non-linear term occurring in Model 1 is $f\left(x_{i j k}^{v r s} / d_{j k s}\right)$ (we call it ( $\left.o_{i j k}^{v r s}\right)$ from now on). A piecewise linear approximation to this function (see Fig. 2) is depicted in Fig. 5. The curve in Fig. 2 is divided into four straight line portions. 


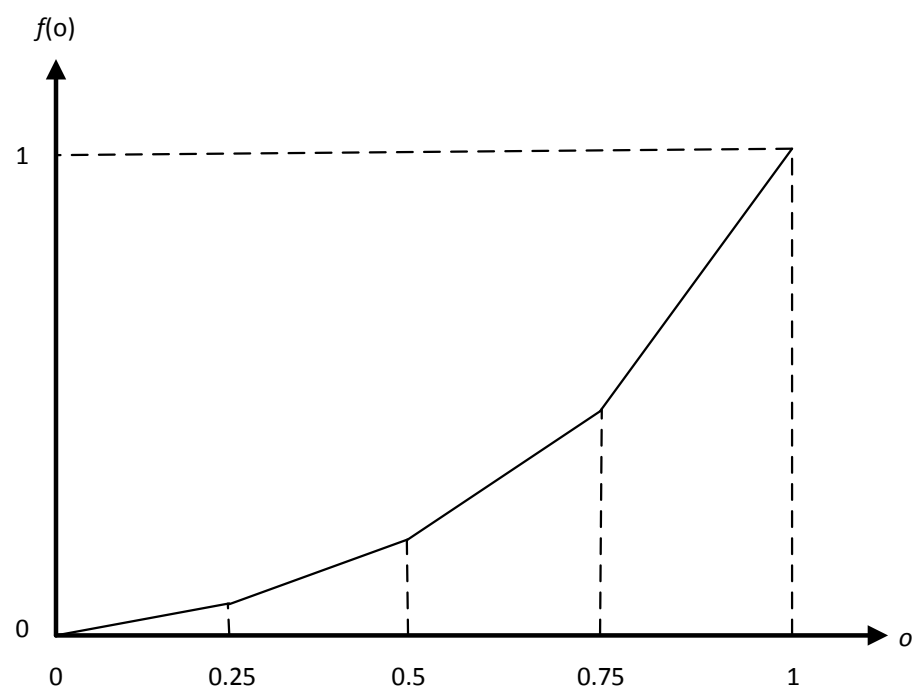

Fig. 5. Piecewise linear utility function for the satisfied demand

The curve in Fig. 5 can be formulated as follows.

$$
f(o)=\left\{\begin{array}{lr}
\frac{4 o}{13}, & o<0.25 \\
\frac{8 o-1}{13}, & 0.25 \leq o<0.5 \\
\frac{16 o-5}{13}, & 0.5 \leq 0<0.75 \\
\frac{24 o-11}{13}, & o \geq 0.75
\end{array}\right.
$$

Our purpose is to eliminate the non-linear term $f\left(o_{i j k}^{v r s}\right)$ from Model 1. This can be done by replacing it by the single linear term $\omega_{i j k}^{v r s}$. Now, it is possible to relate $\omega_{i j k}^{v r s}$ to $o_{i j k}^{v r s}$ by the following relationships.

$$
\begin{aligned}
& o_{i j k}^{v r s}-0 \times \lambda 1_{i j k}^{r r s}+\frac{1}{4} \times \lambda 2_{i j k}^{v r s}+\frac{1}{2} \times \lambda 3_{i j k}^{v r s}+\frac{3}{4} \times \lambda 4_{i j k}^{v r s}+1 \times \lambda 5_{i j k}^{v r s} \quad \forall i, j, k, v, r, s \\
& \omega_{i j k}^{v r s}-0 \times \lambda 1_{i j k}^{v r s}+\frac{1}{13} \times \lambda 2_{i j k}^{v r s}+\frac{3}{13} \times \lambda 3_{i j k}^{v r s}+\frac{7}{13} \times \lambda 4_{i j k}^{v r s}+1 \times \lambda 5_{i j k}^{v r s}=0 \quad \forall i, j, k, v, r, s \\
& \lambda 1_{i j k}^{v r s}+\lambda 2_{i j k}^{v r s}+\lambda 3_{i j k}^{v r s}+\lambda 4_{i j k}^{v r s}+\lambda 5_{i j k}^{v r s}=1 \quad \forall i, j, k, v, r, s
\end{aligned}
$$

$\lambda i$ are new variables introduced into Model 1. They can be interpreted as 'weights' to be attached to the vertices of the curve in Fig. 5. However, it is necessary to add another stipulation regarding $\lambda i$.

At most two adjacent $\lambda i$ can be non-zero 
The stipulation (22) assures that corresponding values of $o_{i j k}^{v r s}$ and $\omega_{i j k}^{v r s}$ lie on one of the straight line segments. For instance, if $\lambda 2=0.5$ and $\lambda 3=0.5$ (other $\lambda i$ are zero), we can get $o=0.375$ and $\omega=0.154$. Obviously, ignoring stipulation (22) will incorrectly allow the possibility of values $o$ and $\omega$ off the piecewise straight lines.

Equations (19) to (21) generates the constraints (34) to (36) that should be added to the original presented model. The term $f\left(x_{i j k}^{v r s} / d_{j k s}\right)$ or $f\left(o_{i j k}^{v r s}\right)$ is replaced by $\omega_{i j k}^{v r s}$. This results in the following Model 2.

$$
\begin{aligned}
& \operatorname{Min} z_{1}=\sum_{i \in I}\left(f_{i} y_{i}+\sum_{k \in K} A C_{k} q_{i k}\right)+\sum_{s \in S} p_{s} \sum_{i \in I} \sum_{k \in K}\left(\sum_{j \in J} \sum_{v \in V} \sum_{r \in A_{i j v}} T C^{v} x_{i j k}^{v r s}+h_{k} z_{i k s}\right) \\
& \operatorname{Max} z_{2}=\sum_{s \in S} \sum_{j \in J} p_{s} \varphi_{j s} \\
& \operatorname{Min} z_{3}=\sum_{s} p_{s} \delta_{s} \\
& \text { s.t. } \\
& x_{i j k}^{v r s} \leq M t_{i j}^{v r s} \quad \forall i \in I, j \in J, v \in V, r \in A_{i j v}, k \in K, s \in S \\
& \sum_{j \in J} \sum_{v \in V} \sum_{r \in A_{i j v}} x_{i j k}^{v r s}+z_{i k s}=\rho_{i k s} q_{i k} \quad \forall i \in I, k \in K, s \in S \\
& w_{j k s}+\sum_{i \in I} \sum_{v \in V} \sum_{r \in A_{i j v}} x_{i j k}^{v r s}=d_{j k s} \quad \forall j \in J, k \in K, s \in S \\
& w_{j k s} \leq \psi_{j k s} d_{j k s} \quad \forall j \in J, k \in K, s \in S \\
& \sum_{i=I} q_{i k} \leq \vartheta_{k} \quad \forall k \in K \\
& q_{i k} \leq y_{i} \gamma_{i k} \quad \forall i \in I, k \in K \\
& \varphi_{j s}=\sum_{i \in I} \sum_{v \in V} \sum_{r \in R} \sum_{k \in K}\left(\tau_{j s} \times \xi_{k} \times \omega_{i j k}^{v r s} \times \Xi\left(t_{i j}^{v r s}\right)\right) \\
& -\delta_{s} \leq \varphi_{p s}-\varphi_{q s} \leq \delta_{s} \quad \forall s, \forall p \neq q \in J \\
& -O_{i j k}^{v r s}+0 \times \lambda 1_{i j k}^{v r s}+\frac{1}{4} \times \lambda 2_{i j k}^{v r s}+\frac{1}{2} \times \lambda 3_{i j k}^{v r s}+\frac{3}{4} \times \lambda 4_{i j k}^{v r s}+1 \times \lambda 5_{i j k}^{v r s}=0 \quad \forall i, j, k, v, r, s \\
& -\omega_{i j k}^{v r s}+0 \times \lambda 1_{i j k}^{v r s}+\frac{1}{13} \times \lambda 2_{i j k}^{v r s}+\frac{3}{13} \times \lambda 3_{i j k}^{v r s}+\frac{7}{13} \times \lambda 4_{i j k}^{v r s}+1 \times \lambda 5_{i j k}^{v r s}=0 \quad \forall i, j, k, v, r, s \\
& \lambda 1_{i j k}^{v r s}+\lambda 2_{i j k}^{v r s}+\lambda 3_{i j k}^{v r s}+\lambda 4_{i j k}^{v r s}+\lambda 5_{i j k}^{v r s}=1 \quad \forall i, j, k, v, r, s \\
& x_{i j k}^{v r s}, q_{i k}, z_{i k s}, w_{j k s}, \varphi_{j s}, \delta_{s}, o_{i j k}^{v r s}, \omega_{i j k}^{v r s}, \lambda 1_{i j k}^{v r s}, \ldots, \lambda 5_{i j k}^{v r s} \geq 0 \quad \forall i, j, v, r, k, s \\
& y_{i} \in\{0,1\} \quad \forall i \in I
\end{aligned}
$$

Stipulation (22) is employed for the set of variables $\lambda i$. In a convex problem, Stipulation (22) will be satisfied automatically (i.e. original separable programming is sufficient). However, in a non-convex problem, as our problem is, this stipulation cannot be modeled 
without adding new binary variables and logical constraints. According to Williams (2013), this restriction does not need to be modeled explicitly. Instead, it can be said that the set of variables $\left(\lambda_{1}, \lambda_{2}, \ldots, \lambda_{n}\right)$ is a Specially Ordered Set variables of type 2 (SOS2). At most two variables within the SOS2 can take on non-zero values. The two non-zero values have to be for adjacent variables in that set (cf. Williams (2013); p. 177-182). Hence, this type of variable definition is used in our case and coded in General Algebraic Modeling System (GAMS) software.

Noteworthy, the applied formulation method in our case is usually known as the $\lambda$ form for separable programming where variables $\lambda i$ denote as weights attached to the vertices in the piecewise straight line.

\subsection{Augmented $\varepsilon$-constraint method}

In the traditional $\varepsilon$-constraint method, the most important objective function (i.e., the second objective in this paper that is selected with regard to the decision-maker opinion) is optimized while the other objectives (i.e., the first and third objective functions) are transformed into constraints as follows.

\section{Max OF2}

s.t.

$$
\begin{aligned}
& \text { OF1 } \leq \varepsilon_{1} \\
& \text { OF3 } \leq \varepsilon 3
\end{aligned}
$$

Constraints (26) to (38)

Then, the Pareto-optimal (i.e. efficient) solutions of the model are achieved by parametrical variation in the right hand side (i.e. $\varepsilon_{1}$ and $\varepsilon_{3}$ ) of the constrained objective functions (Mavrotas, 2009; Azadeh et al., 2015). The range of $\varepsilon_{1}$ and $\varepsilon_{3}$ can be calculated by optimizing the constrained objective functions OF1 and OF3 separately subject to the constraints and constructing the pay-off table. After that, different values for $\varepsilon_{2}$ and $\varepsilon_{3}$ can be calculated by dividing the range of constrained objectives OF1 and OF3 (i.e. $r_{1}$ and $r_{3}$ ) to $q$ equal intervals as follows.

$$
\begin{aligned}
& r_{1}=\mathrm{OF} 1^{\mathrm{max}}-\mathrm{OF} 1^{\mathrm{min}} ; \quad \varepsilon_{1}^{l}=\mathrm{OF} 1^{\max }-\frac{r_{1}}{q} \times l \quad l=0, \ldots, q-1 \\
& r_{3}=0 F 3^{\max }-0 F 3^{\min } ; \quad \varepsilon_{3}^{l}=0 F 3^{\max }-\frac{r_{3}}{q} \times l \quad l=0, \ldots, q-1
\end{aligned}
$$

However, the general form of the $\varepsilon$-constraint method has some disadvantages. For instance, this method does not assure efficiency of the achieved solutions (i.e. obtaining weakly efficient solutions) (Mavrotas, 2009). Mavrotas (2009) expressed some of these disadvantages and developed an improved version of the $\varepsilon$-constraint method, namely "augmented $\varepsilon$-constraint method". The formulation of the augmented $\varepsilon$-constraint method for our problem is as follows. 
$\operatorname{Max} \mathrm{OF} 2+\left(\varphi_{1} \times s_{1}\right)+\left(\varphi_{3} \times s_{3}\right)$

s.t.

$$
\begin{aligned}
& \text { OF1 }-s_{1}=\varepsilon_{1} \\
& \text { OF3 }-s_{3}=\varepsilon_{3} \\
& \text { Constraints (26)-(38) } \\
& s_{1}, s_{3}>0
\end{aligned}
$$

where $\varphi_{1}$ and $\varphi_{3}$ are sufficient small numbers (usually between $10^{-3}$ and $10^{-6}$ ) and augmented terms $\varphi_{1} \times s_{1}$ and $\varphi_{3} \times s_{3}$ assure obtaining just an efficient solution for each epsilon vector. This method is then applied to solve the multi-objective model in order to achieve efficient solutions.

\subsection{PROMETHEE-II}

PROMETHEE is one of the most appropriate Multi-Criteria Decision-Making (MCDM) techniques that can yield full ranking of alternatives (i.e. Pareto optimal solutions). Solutions are first evaluated by different evaluation criteria (i.e. values of objective functions), and then a definite preference function, $p_{j}(a, b)$, is selected with regard to each evaluation criteria $j$ to calculate the relative preference of each solution over other ones. The preference function is defined by Equation (43), where $G_{j}$ is a non-decreasing function of the obtained deviation $(d)$ between the performances of the solutions $a$ and $b$ $\left(f_{j}(a)-f_{j}(b)\right)$ using the considered evaluation criteria $j$.

$$
P_{j}(a, b)=G_{j}\left\{f_{j}(a)-f_{j}(b)\right\}
$$

There are six general shapes for such non-decreasing functions, among them a specific function is selected for each criterion, according to their nature and characteristics. Type $V$ preference function is selected for our problem. It is defined by Equation (44), where $q$ is a difference threshold and $p$ is a strict preference threshold (Tzeng and Huang, 2011):

$$
\begin{aligned}
& P(a, b)=0 \text { for } d \leq q \\
& P(a, b)=\frac{d-q}{p-q} \text { for } q \leq d \leq p \\
& P(a, b)=1 \text { otherwise }
\end{aligned}
$$

The overall preference index, $\pi(a, b)$, is computed by Equation (45), where $w_{j}$ denotes the importance weight of criterion $j$ obtained based on the opinion of the decision-maker (DM). Positive $\varphi^{+}(a)$ and negative $\varphi^{-}(a)$ preference flows, which measure how an solution $(a)$ outranks or is outranked by the other solutions, are calculated by Equations (46) and (47), respectively. The difference between these preference flows is represented as the net preference flow $\varphi(a)$ (see Equation (48)). A higher value of $\varphi(a)$ reflects a higher attractiveness of solution $a$ (Turcksina et al., 2011). 


$$
\begin{aligned}
& \pi(a, b)=\sum_{j=1}^{k} w_{j} P_{j}(a, b) \\
& \varphi^{+}(a)=\sum_{b}^{b} \pi(a, b) \\
& \varphi^{-}(a)=\sum_{b} \pi(b, a) \\
& \varphi(a)=\varphi^{+}(a)-\varphi^{-}(a)
\end{aligned}
$$

Finally, solutions are ranked from the best to the worst one by using the net flow vector $(\varphi)$ in PROMETHEE-II to find a complete ranking of solutions.

The augmented $\varepsilon$-constraint method presents some Pareto-optimal solutions. However, the DM of the considered case study needs a single solution to construct the HRL network. Therefore, the DM is requested to state his/her preference about the objective functions regarding the managerial policy of this case study (see the weights, $w$, in Table 4). Regarding the DM's preference, the best Pareto-optimal solution can be determined by the PROMETHEE-II method (see the highlighted row in Table 4).

As can be derived, the best Pareto-optimal solution is changed by considering different preferences. So, the best solution highly depends on the DM's opinion.

\section{Computational experiments}

In order to display the applicability of the presented model and methods, the augmented $\varepsilon$-constraint method is coded in the GAMS software and integrated into Model 2 to solve a randomly generated test problem (i.e., $|I| \times|J| \times|K| \times|V| \times|R| \times|S|=5 \times 3 \times 2 \times$ $2 \times 2 \times 8$ ). The values of the parameters are generated from the probability distributions listed in Table 2. GAMS (ver. 24.1.2) using the CPLEX solver (ver. 12.5.1.0) is able to solve MILP models. The PC with Intel® Core ${ }^{\mathrm{TM}}$ i5-760 processor, $2.8 \mathrm{GHz}, 12 \mathrm{~GB}$ of RAM, and the Windows 7 operating system is used as a technical platform.

\section{Table 2}

Data of the test problems

\begin{tabular}{ccc}
\hline$f_{i}$ & $A C_{k}$ & $\gamma_{i k}$ \\
\hline$\sim \mathrm{U}(10 \mathrm{E}+8,105 \mathrm{E}+10)$ & $\sim \mathrm{U}(0.9 \mathrm{E}+2,1.1 \mathrm{E}+2)$ & $\sim \mathrm{U}(2.1 \mathrm{E}+8,2.1 \mathrm{E}+10)$ \\
\hline$h_{k}$ & $\vartheta_{k}$ & $p_{s}, \rho_{i k s}, \tau_{j s}, \xi_{k}, \psi_{j k s}$ \\
\hline$\sim \mathrm{U}(10 \mathrm{E}+2,10 \mathrm{E}+4)$ & $\sim \mathrm{U}(2.1 \mathrm{E}+10,2.1 \mathrm{E}+14)$ & $\sim \mathrm{U}(0,1)$ \\
\hline$t_{i j}^{\text {vrs }}$ & $T C_{i j k}^{\text {vrs }}$ & $d_{j k s}$ \\
\hline$\sim \mathrm{U}(1,100)$ & $\sim \mathrm{U}(10 \mathrm{E}+3,10 \mathrm{E}+5)$ & $\sim \mathrm{U}(10 \mathrm{E}+5,10 \mathrm{E}+7)$ \\
\hline
\end{tabular}

The obtained configuration of the network for the test problem using different objective functions are shown in Figs. 6 to 8. When we consider OF1 to be minimized (see Fig. 6), Warehouses \#1 and \#2 are opened to distribute RCs to all the five DPs through airways. Fig. 7 also shows that the DPs are served by Warehouses \#1-\#3 through a mixture of airways and overland route when OF2 is maximized. When OF3 is selected to 
be minimized, a complex transportation system of airways and overland routes is employed for the distribution of RCs (see Fig. 8). These imply the effect of the different objective functions on the configuration of the network. In addition, Fig. 9a-b depict the values of the objective functions of the Pareto-optimal solutions. It can be concluded that the results from employing different objective functions are not necessarily consistent, and therefore the objective functions should be considered separately.
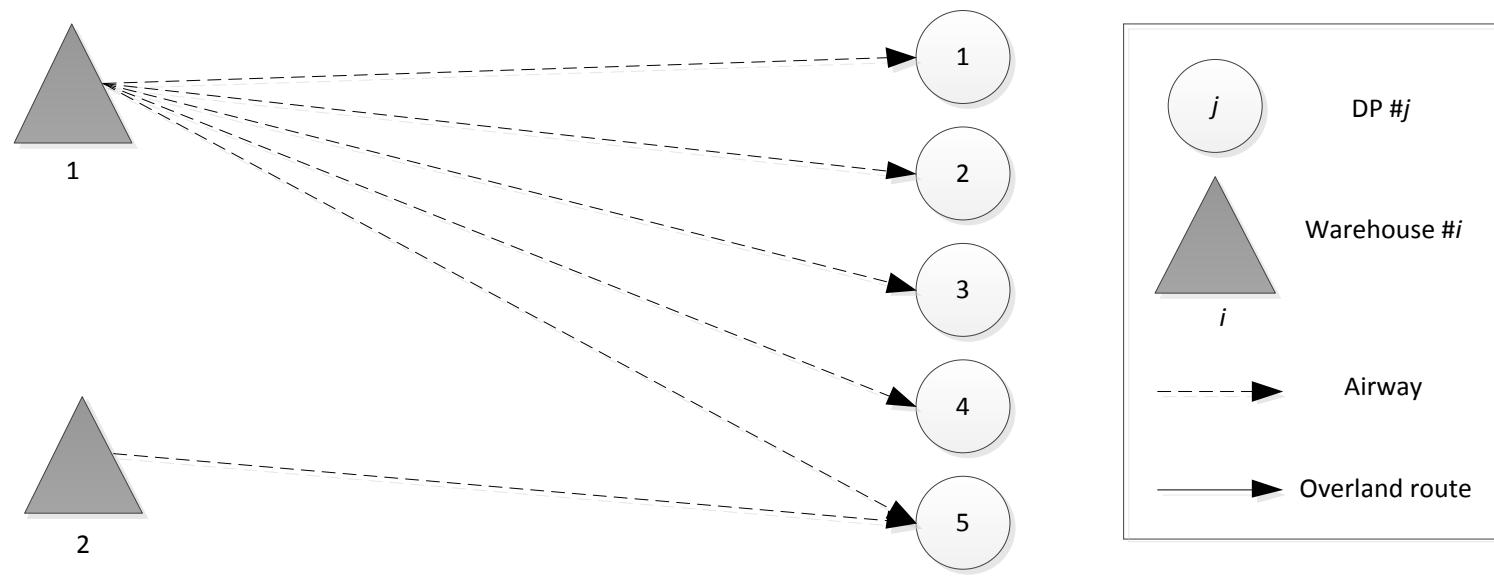

Fig. 6. Obtained network configuration by considering OF1 to be minimized

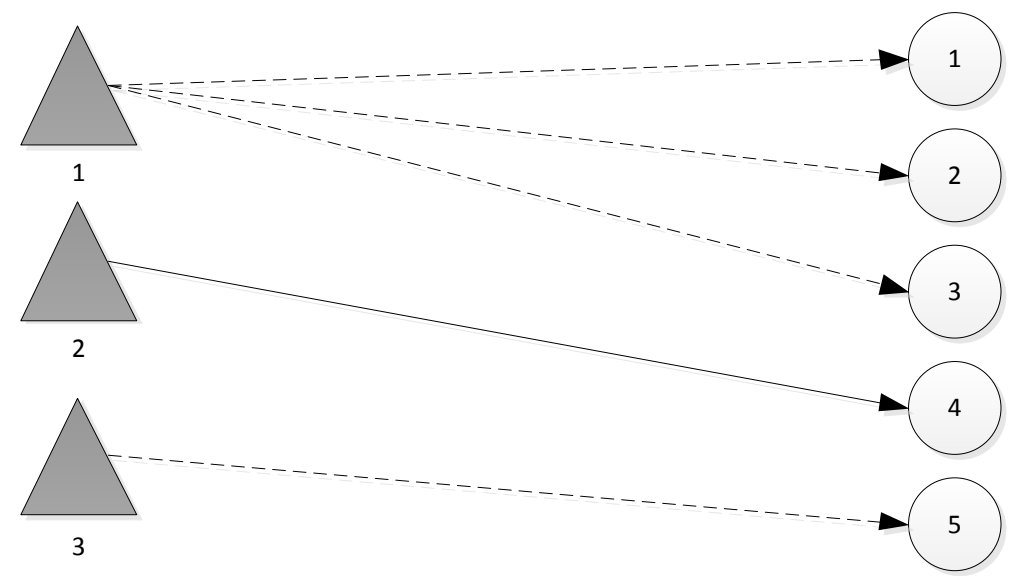

Fig. 7. Obtained network configuration by considering OF2 to be maximized

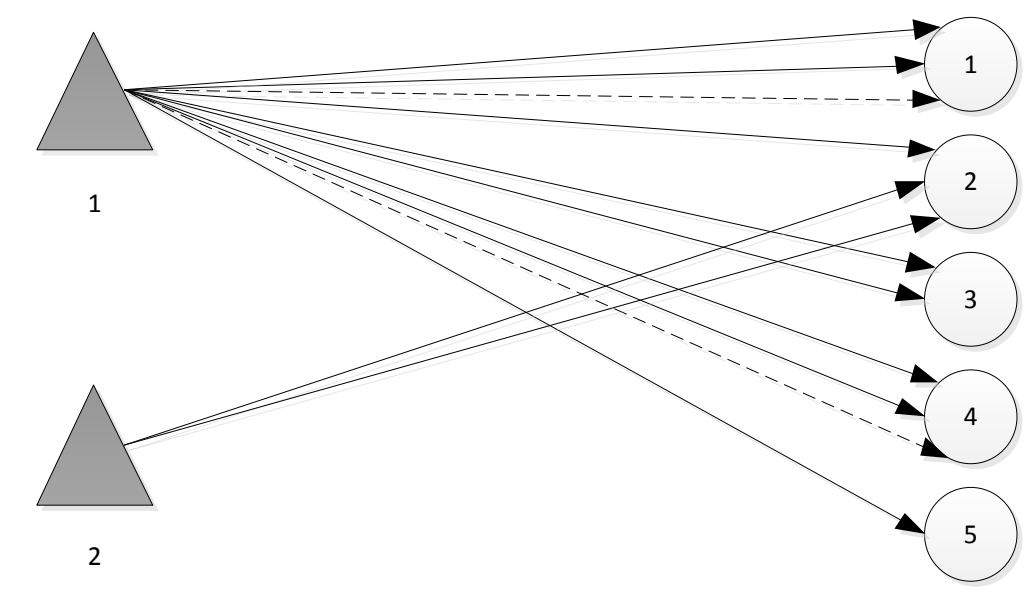


Fig. 8. Obtained network configuration by considering OF3 to be minimized

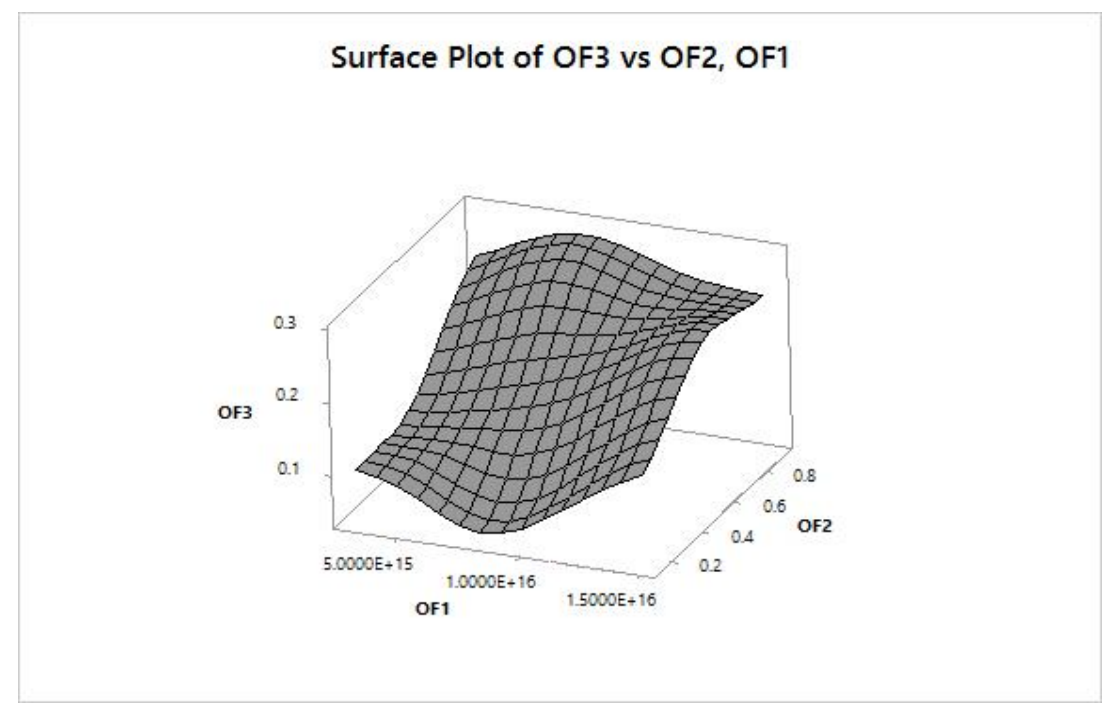

(a)

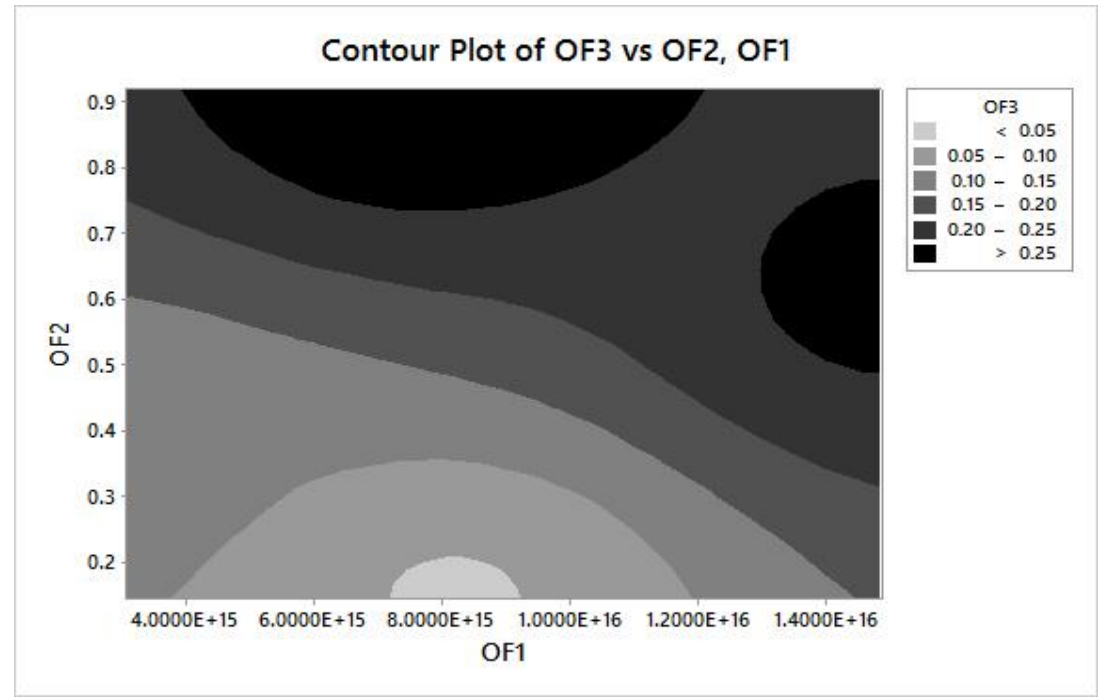

(b)

Fig. 9. Pareto frontier; (a) Surface plot, (b) Contour plot

\subsection{Sensitivity analysis}

To show the sensitivity of the model with respect to the important parameters used in the $M R$ utility level (i.e., $\tau_{j s}$ and $\xi_{k}$ ), we introduce four indices (see Equations (49)-(52)). Equation (49) measures the Expected Time-Weighted Demand Satisfaction for DP $j$ $\left(E T W D S_{j}\right)$ and reflects the time-dependent attention of the network to DP $j$. Equation (50) measures the Expected Satisfied Demand Ratio for DP $j\left(E S D R_{j}\right)$ and shows the demanddependent attention of the network to DP $j$. The Expected Time-Weighted Delivered Amount of RC $k\left(E T W D A_{k}\right)$ is measured by Equation (51). This index represents the importance of RC $k$ for a timely distribution in the network. Equation (52) addresses the Expected Delivered Amount of RC $k\left(E D A_{k}\right)$. This index helps investigate the expected delivered amount of RC $k$ with regard to its priority in the network. 


$$
\begin{aligned}
& E T W D S_{j}=\sum_{s} p_{s} \sum_{i} \sum_{k} \sum_{v} \sum_{r} t_{i j k}^{v r s} x_{i j k}^{v r s} \\
& E S D R_{j}=\sum_{s} p_{s} \sum_{i} \sum_{k} \sum_{v} \sum_{r} \frac{x_{i j k}^{v r s}}{d_{j k s}} \\
& E T W D A_{k}=\sum_{s} p_{s} \sum_{i} \sum_{j} \sum_{v} \sum_{r} t_{i j}^{v r s} x_{i j k}^{v r s} \\
& E D A_{k}=\sum_{s} p_{s} \sum_{i} \sum_{j} \sum_{v} \sum_{r} x_{i j k}^{v r s}
\end{aligned}
$$

Fig. 10a-b are some examples to show the influence of the increase in the priority of DP 4 on $E T W D S_{4}$ and ESDR 4 , respectively. Fig. 10a shows that an increase in the priority of DP 4 decreases ETWDS 4 when OF2 is maximized; however, it does not influence the value of ETWDS 4 because the priority parameter is not included in OF1. Any absolute trend is not seen in ETWDS 4 when OF3 is minimized, because this objective just attempts to establish a balance among DPs and the model may be forced to satisfy demand amount of DP 4 in longer or shorter duration times. Indeed, when OF2 is maximized, ETWDS 4 decreases because the model attempts to satisfy the demand of the high-prioritized DPs in less time.

In a similar way, the evolution of $E S D R_{4}$ when different objective functions are considered is viewed in Fig. 10b. The increase in ESDR 4 is due to the increase of demand satisfaction for the high-prioritized DPs. Although we note a constant value after the second point (i.e., follow the red line in Fig. 10b), the demand of DP 4 is completely satisfied and the model continuously decreases ETWDS 4 (i.e., follow the red line in Fig. 10a). The model is continuously increasing the utility of the delivered RCs to DP 4, while its priority is increasing.

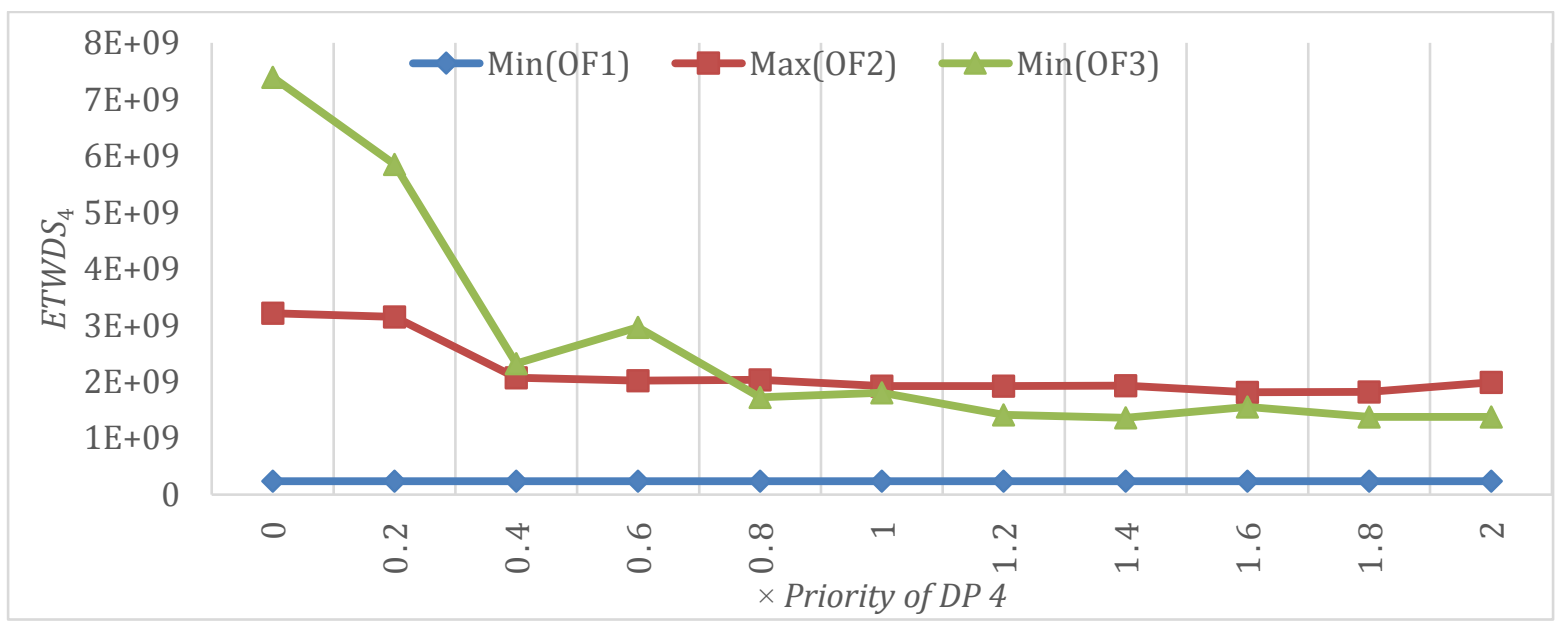

(a) 


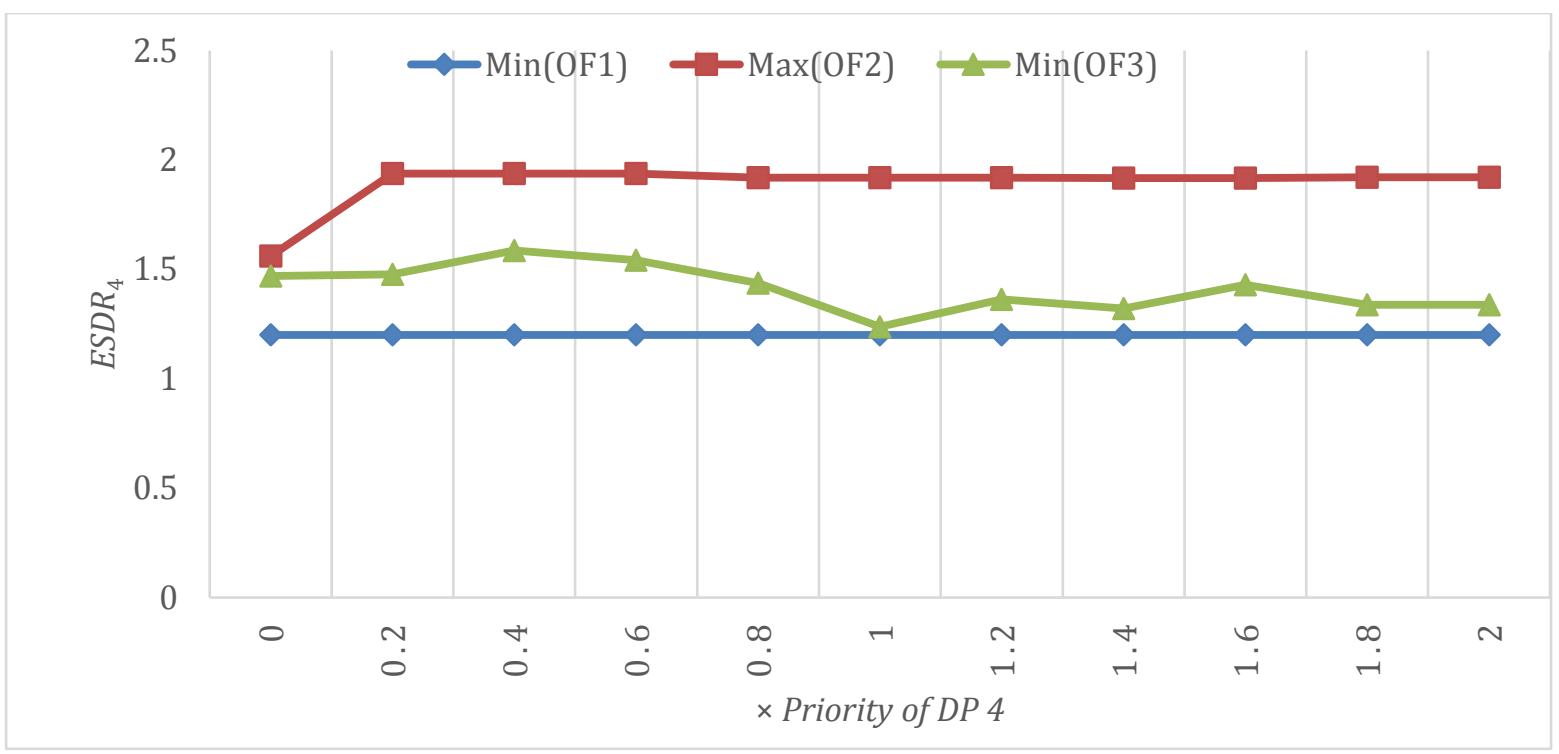

(b)

Fig. 10. (a) Behavior of the $E T W D S_{4}$ amount vs. the changes of the priority of DP $4\left(\tau_{4 s}\right)$ and

(b) Behavior of the ESDR 4 amount vs. the changes of $\tau_{4 s}$

Fig. 11a-b illustrate the impact of the increase in the priority of RC 1 on ETWDA 1 and $E D A_{1}$, respectively. Fig. 11a shows that an increase in the priority of RC 1 does not influence $E T W D A 1$ when OF1 is minimized, because the priority parameter is not included in this objective function. In addition, it can be concluded that $E T W D A_{1}$ is impressible by variation of $\xi_{1}$ when OF3 is minimized; however, there is no any absolute trend in its changes. ETWDA 1 decreases because the model tries to satisfy the high-prioritized RC 1 in less time when OF2 is maximized. In the similar way, Fig. 11b can be analysed. Because the priority parameter is not included in $\mathrm{OF} 1, E D A_{1}$ is not impressible by the variation of $\xi_{1}$ when this objective function is minimized. Since OF3 just tries to establish a balance among DPs, the model may be forced to satisfy lower or higher amount of demand in the different cases and therefore $E D A_{1}$ is impressible by the variation of $\xi_{1}$. $E D A_{1}$ is improved by an increase of $\xi_{1}$ when OF2 is maximized. This happens because the model tries to deliver the high-prioritized RC 1 in a larger amount. Although, we see a constant behaviour after the second point (i.e., follow the red line in Fig. 11a), the model continuously tries to increase $E D A_{1}$ (i.e., follow the red line in Fig. 11b). This means that the model is continuously trying to increase the utility of delivered RC 1 when its priority is increasing. 


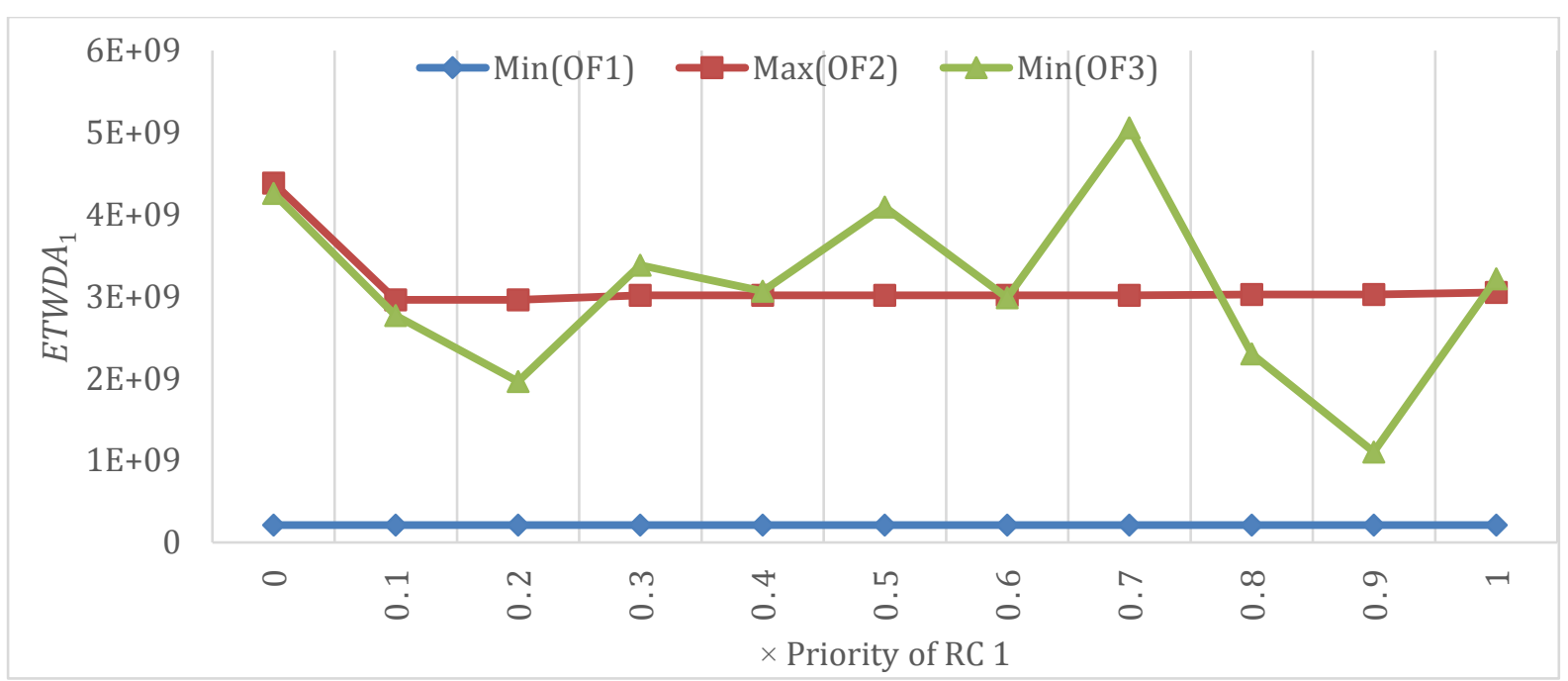

(a)

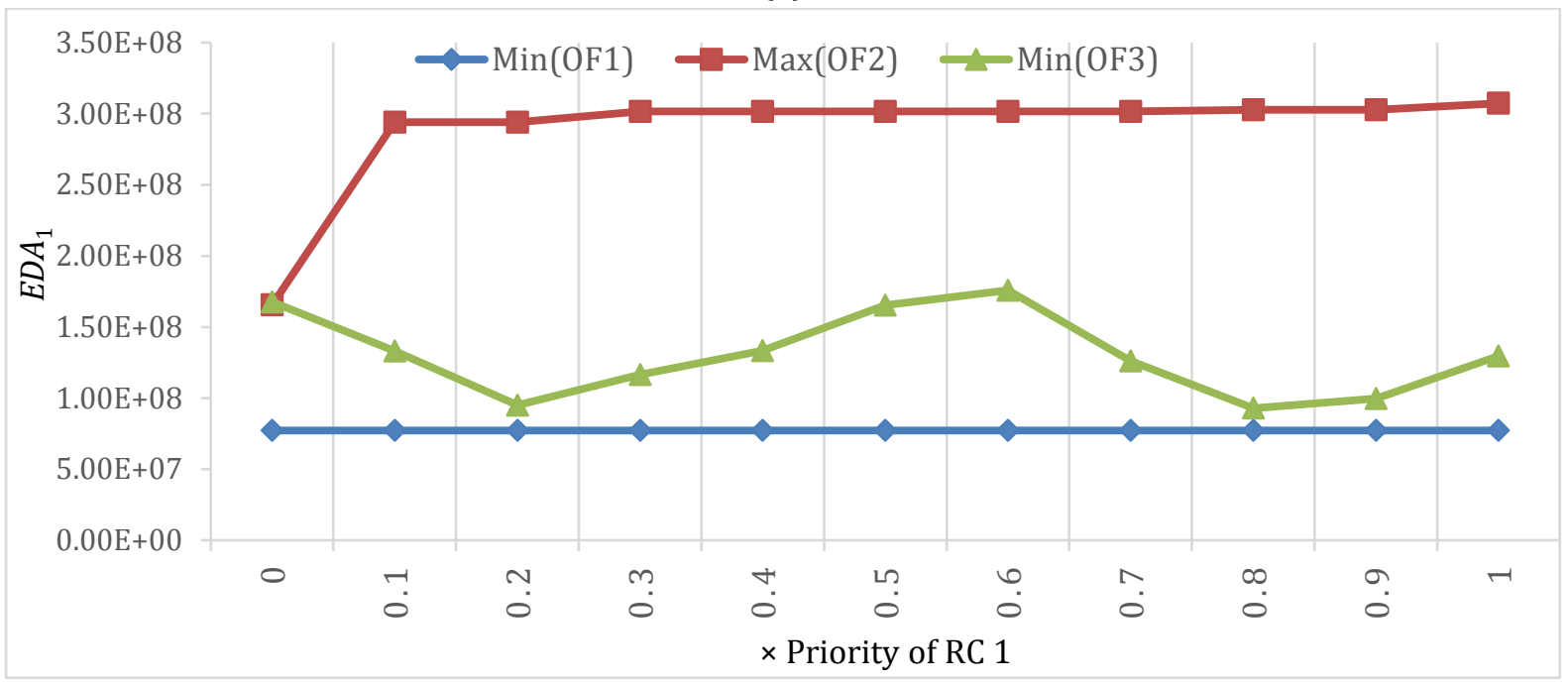

(b)

Fig. 11. (a) Behavior of the $E T W D A_{1}$ amount vs. the changes of priority of RC $1\left(\xi_{1}\right)$ and (b) Behavior of the $E D A_{1}$ amount vs. the changes of $\xi_{1}$

\subsection{A benchmark study}

To be more confident about the presented solution approach (i.e. the integrated separable programming-augmented $\varepsilon$-constraint method), a comparison with the NSGAII algorithm, that is well-known and efficient for solving the MINLP models, is provided in this section. The NSGA-II algorithm is coded in the MATLAB software (ver. R2011a) and integrated into Model 1. Eight test problems are considered in the different sizes (see Table 3). The values of the parameters are generated from the probability distributions listed in Table 2. Table 4 provides the considered values of the parameters of NSGA-II.

\section{Table 3}

Considered sizes for the test problems 


\begin{tabular}{|c|c|}
\hline Problem number & $|I| \times|J| \times|K| \times|V||\times| R|| \times|S|$ \\
\hline 1 & $2 \times 6 \times 2 \times 1 \times 1 \times 2$ \\
\hline 2 & $3 \times 8 \times 2 \times 1 \times 1 \times 2$ \\
\hline 3 & $3 \times 10 \times 2 \times 2 \times 1 \times 3$ \\
\hline 4 & $4 \times 12 \times 3 \times 2 \times 1 \times 4$ \\
\hline 5 & $4 \times 14 \times 3 \times 1 \times 2 \times 5$ \\
\hline 6 & $5 \times 16 \times 3 \times 2 \times 2 \times 5$ \\
\hline 7 & $5 \times 18 \times 4 \times 2 \times 2 \times 6$ \\
\hline 8 & $6 \times 22 \times 5 \times 2 \times 2 \times 8$ \\
\hline
\end{tabular}

Table 4

Parameters setting for NSGA-II

\begin{tabular}{lrr}
\hline Parameter & & Value \\
\hline Number of iterations & & 250 \\
Pop size & & 100 \\
Maximum number of non-dominated solutions & & 100 \\
Cross over probability & & 0.3 \\
Mutation probability & & 0.2 \\
\hline
\end{tabular}

NSGA-II and the integrated separable programming-augmented $\varepsilon$-constraint (ISPAE) method are compared with each other by some well-known metrics. The metrics used for measurement of the performance of the algorithms are as follows: the number of Paretooptimal solutions, CPU time, quality of Pareto-optimal solutions, and the diversity of them. The readers can refer to Rezaei-Malek et al. (2016) for more information about these metrics.

Table 5 represents the differences of NSGA-II and ISPAE with regard to the required CPU time and the obtained number of Pareto solutions. Although NSGA-II obtained more solutions than the ISPAE method, it is not capable to solve the large-sized problems in a reasonable time. Table 6 shows the differences of the two methods with regard to the diversity and standard deviation of the obtained Pareto solutions. As can be derived, none of the algorithms has an absolute advantage over the other. Moreover, there is no difference between the qualities of the obtained Pareto-optimal solutions by both of the algorithms.

Table 5

Solution properties

\begin{tabular}{|c|c|c|c|c|}
\hline \multirow{2}{*}{ Test problem } & \multicolumn{2}{|c|}{ CPU time (seconds) } & \multicolumn{2}{|c|}{ Number of Pareto solutions } \\
\hline & NSGA-II & ISPAE & NSGA-II & ISPAE \\
\hline 1 & 1059.161 & $29.8283^{*}$ & 99 & 35 \\
\hline 2 & 1678.089 & 31.4671 & 99 & 45 \\
\hline 3 & $5.13 \mathrm{E}+03$ & 65.3281 & 92 & 37 \\
\hline 4 & 15035.58 & 99.2159 & 70 & 42 \\
\hline 5 & 21618.59 & 179.166 & 92 & 20 \\
\hline 6 & $>50000$ & 217.682 & NA & 12 \\
\hline 7 & $>50000$ & 1861.07 & NA & 17 \\
\hline 8 & $>50000$ & 16770.8 & NA & 27 \\
\hline
\end{tabular}

* The bold numbers are the better results 
Table 6

Diversity and standard deviation of Pareto solutions

\begin{tabular}{|c|c|c|c|c|}
\hline \multirow{2}{*}{ Test problem } & \multicolumn{2}{|c|}{ Diversity } & \multicolumn{2}{|c|}{ Standard deviation } \\
\hline & NSGA-II & ISPAE & NSGA-II & ISPAE \\
\hline 1 & $5.9561 \mathrm{E}+14$ & $2.9578 E+14$ & $1.99578 \mathrm{E}+12$ & $3.23181 \mathrm{E}+12$ \\
\hline 2 & $5.2292 \mathrm{E}+14$ & $3.2909 \mathrm{E}+14$ & $1.9291 \mathrm{E}+13$ & $3.96666 \mathrm{E}+12$ \\
\hline 3 & $6.4554 \mathrm{E}+14$ & $4.6547 \mathrm{E}+14$ & $8.3192 \mathrm{E}+12$ & $6.11238 E+12$ \\
\hline 4 & $3.033 E+14$ & $5.7834 \mathrm{E}+14$ & $3.2749 \mathrm{E}+12$ & $6.07203 E+12$ \\
\hline 5 & $1.0264 \mathrm{E}+15$ & $1.2863 E+15$ & 7.34145E+12 & $2.67452 \mathrm{E}+13$ \\
\hline 6 & NA & $1.3731 E+15$ & NA & $7.5548 E+13$ \\
\hline 7 & NA & $1.7517 \mathrm{E}+15$ & NA & $3.73126 \mathrm{E}+13$ \\
\hline 8 & NA & $2.2261 \mathrm{E}+15$ & NA & $4.02436 E+13$ \\
\hline
\end{tabular}

* The bold numbers are the better results

To investigate the differences of the obtained Pareto-optimal solutions by these two algorithms, we randomly selected some more similar solutions and explored their differences. We noticed that although there are some minor differences with regard to the amount of shipped RCs by the different mode-route pairs, there is no major difference between solutions, e.g. the decisions about the warehouses and the amount of RCs that should be prepositioned.

According to the above discussion, we can conclude that the obtained solutions by ISPAE are reliable, and also this method is strongly recommended for the large-sized problems.

\section{A real case study: Potential earthquake in Tehran}

Tehran city is the capital of Iran with around 10 million people are daily living or commuting. The history of the region shows the strong earthquakes (i.e. 7.0 Richter and higher) over the 158 last years (Tofighi, 2011). Tehran has been built over the several faults (see Fig. 12). According to the report of Japan International Cooperation Agency (JICA), three faults namely Mosha-Fasham Fault (MFF) and North Tehran Fault (NTF), in the north of Tehran, and Rey Fault (RF) in the south of Tehran are the active ones (JICA, 2010). The JICA also proposed a Hybrid Model (HM) that is a combination of all the faults' activities. 


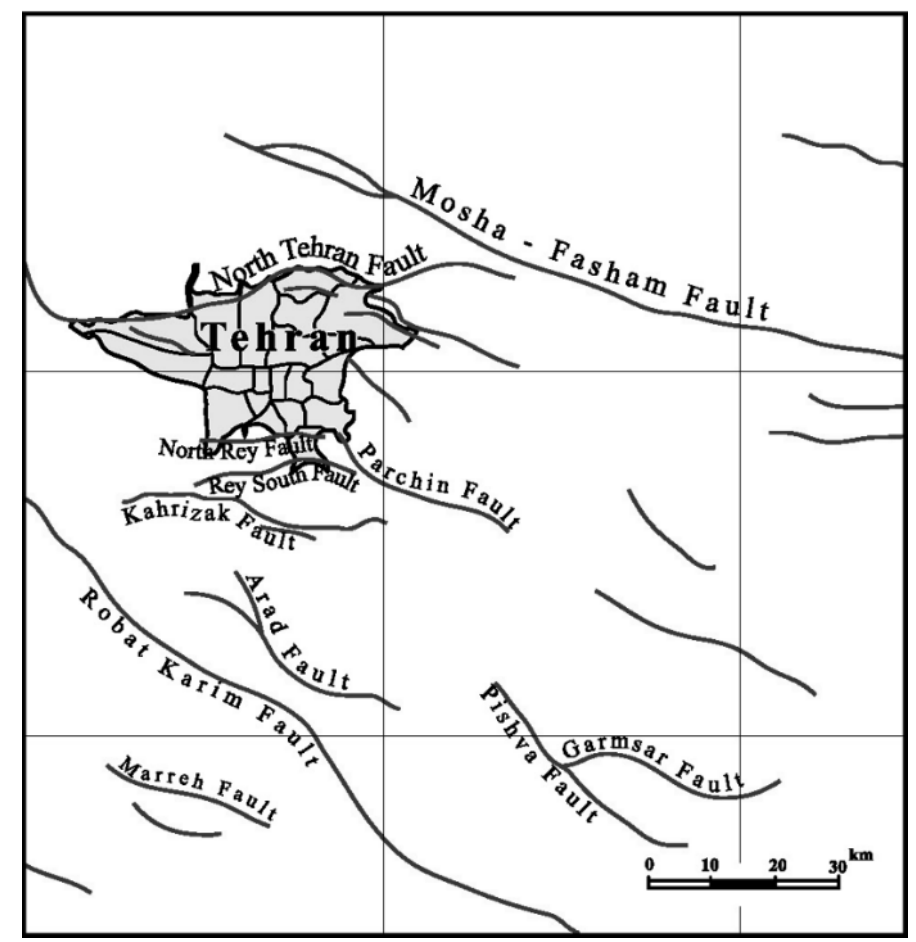

Fig. 12. Fault traces in the Tehran region adopted from www.atlas.tehran.ir

RF can cause a 9.0 Richter earthquake in the southern part and a 7.0-8.0 Richter in the northern part of Tehran. Also, NTF causes an earthquake in the northern part (i.e., almost 9.0 Richter) stronger than in the southern part (i.e., almost 7.0 Richter). MFF can result in at most a 7.0 Richter earthquake. Moreover, it is assumed that HM causes an 8.0-9.0 Richter earthquake throughout Tehran (JICA, 2010).

Tehran city includes 22 sub-divisions that their centres are assumed as DPs (see Fig. 13). The demand of each DP is a population-dependent parameter and is varying during a day. For instance, the demand/population of the sub-division \#12 in non-working hours (NW) (4 PM to $8 \mathrm{AM}$ ) is lower than that of working hours (W) (8 AM to $4 \mathrm{PM}$ ) because this district is a working area.

We need to be able for serving the DPs with respect to 8 different scenarios in terms of the causative faults (MFF, NTF, RF and HM) and the time periods (W and NW). Table 7 shows these different scenarios and their occurrence probabilities as they are estimated in (JICA, 2010).

\section{Table 7}

Eight different scenarios of a plausible earthquake in Tehran adapted from JICA (2010)

\begin{tabular}{cccccccccc}
\hline & \multicolumn{3}{c}{ RF } & \multicolumn{2}{c}{ NTF } & \multicolumn{2}{c}{ MFF } & \multicolumn{2}{c}{ HM } \\
\cline { 2 - 10 } Scenario number & W & NW & W & NW & W & NW & W & NW \\
\cline { 2 - 10 } Probability & $\# 1$ & $\# 2$ & $\# 3$ & $\# 4$ & $\# 5$ & $\# 6$ & $\# 7$ & $\# 8$ \\
\cline { 2 - 10 } & 0.125 & 0.125 & 0.125 & 0.125 & 0.1 & 0.1 & 0.15 & 0.15 \\
\hline
\end{tabular}




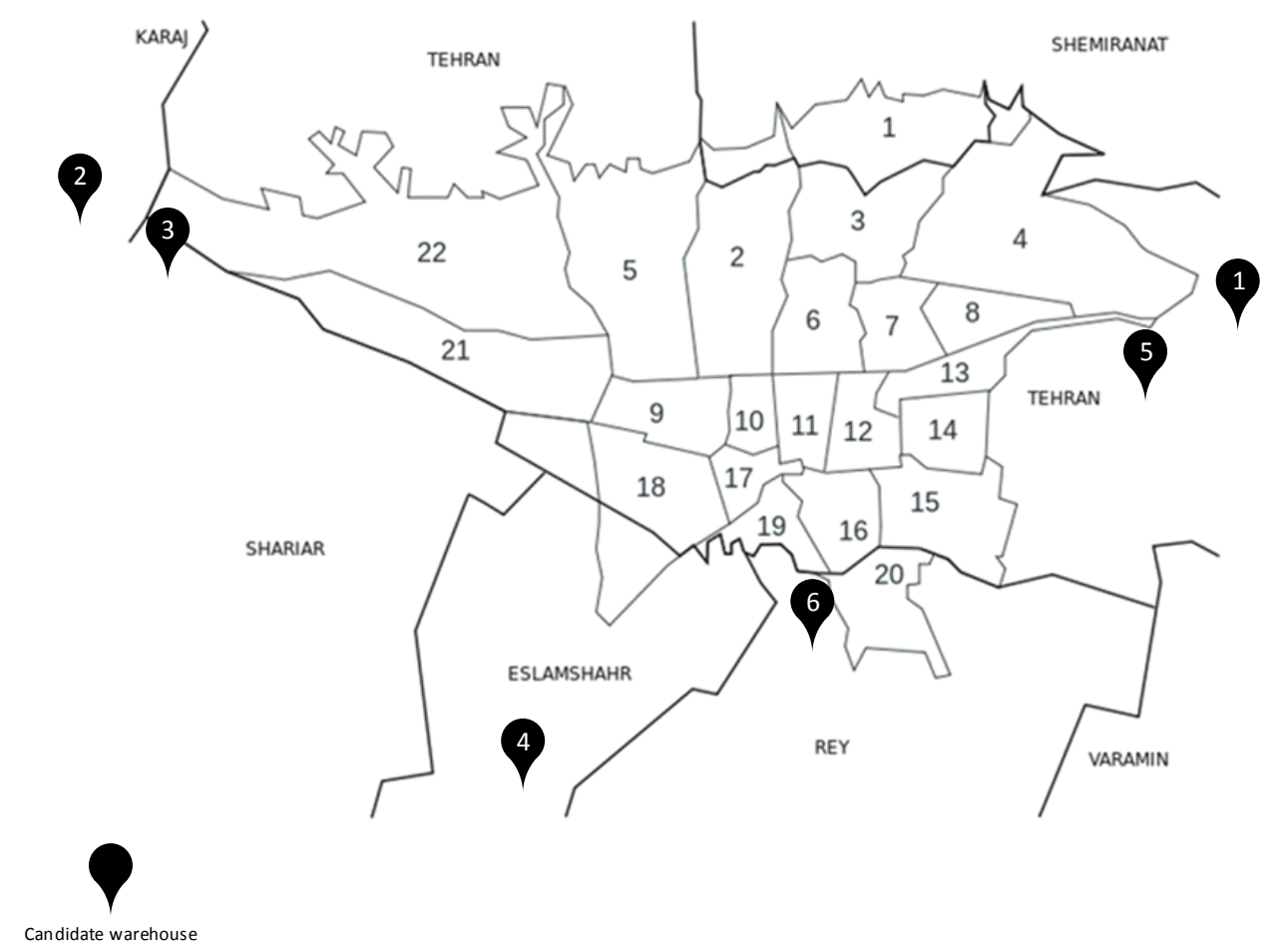

Fig. 13. Map of the Tehran city and the positions of the candidate warehouses

This case study includes six candidate warehouses as shown by the black balloons in Fig. 13, 22 DPs, and the five different types of RC (i.e., non-perishable food package, bottle water, medical kit, blanket, and tent). The mentioned candidate set is determined by the HRL organization with regard to five the criteria, i.e. cost, technical, risk, availability and coverage, as discussed in (Bozorgi-Amiri and Asvadi, 2015).

The priority of the DPs (i.e., 22 sub-divisions) is calculated regarding to 14 vulnerability criteria, i.e. the distance from causative faults, landslide, liquefaction, amplification, downfall, structural behavior of buildings, electricity network, gas network, transportation network, water network, medical emergency services, fire stations, income level of people, search and rescue capability) as proposed by Nateghi (2001). Moreover, according to experts' opinions about an earthquake condition, the priorities of the five different RCs can be determined. The priority of the non-perishable food package, bottle water, medical kit, blanket, and tent are respectively considered as follows: $0.1,0.3,0.5,0.05$ and 0.05 . These weights are adopted from the work of Gralla et al. (2014).

The establishment cost of each warehouse in the six candidate locations is estimated $1.25 \mathrm{e}+10$ Rials, where each one is capable to stock the five types of RC required for 50000 families. The effect of an earthquake, according to the different scenarios, on the warehouses is included in the estimation of JICA (2010) about the proportion of the prepositioned RCs remaining usable at each warehouse with regard to the different criteria (e.g., structure type of surrounding buildings and warehouse, risk of utilities, etc.) (see Supplementary Material).

The demand of the DPs depends on the population size and on the intensity of the earthquake. The demand of the 22 DPs regarding the considered scenarios (see Table 7) 
is estimated based on the population information of the DPs in the different times of a day and the destruction intensity of the earthquake (see Supplementary Material).

Two different types of vehicle (i.e., truck and helicopter) are available. For a truck, two possible overland routes are determined between each warehouse-DP pair. For a helicopter, the shortest airway is considered. Table 8 illustrates the characteristics of the vehicles.

\section{Table 8}

Characteristics of the employed vehicles

\begin{tabular}{ccc}
\hline & Speed $(\mathrm{km} / \mathrm{h})$ & Cost per hour (Rial) \\
\cline { 2 - 3 } Helicopter CH-147D Chinook & 220 & $2.4 \mathrm{E}+6$ \\
Truck HLVW-Cargo & 85 & $0.7 \mathrm{E}+6$ \\
\hline
\end{tabular}

According to the different scenarios, the required time to deliver the RCs to the DPs is estimated by JICA (2010) while considering the different criteria, i.e. the routes' width, structure type of surrounding buildings, traffic, etc. (e.g., considered by Mete and Zabinsky (2010)).

The required budget of the HRL organization, funded by the municipality of Tehran, needs to be minimized along with maximization of the network efficacy and balance.

Table 9 shows the obtained Pareto-optimal solutions by the ISPAE method. The best values of $\varphi_{1}$ and $\varphi_{2}$ are obtained experimentally where these values result in the high quality Pareto optimal solutions. The decision maker (DM) of the case study must select one of these solutions to construct the network. Hence, the PROMETHEE-II method is employed to assist the DM in selecting. Table 9 shows 13 alternatives (i.e., the obtained Pareto-optimal solutions) and three criteria (i.e., values of OF1, OF2 and OF3). The values of $p, q$, and $w$ are determined for each criterion $j$ with regard to the DM's opinion (see Table 10). Table 11 also shows the ranking of the alternatives. The DM selects the first ranked alternative, where all the six warehouses are opened and a mixture of different modes and routes are employed for the distribution of the RCs. Table 12 also shows the quantity of the prepositioned RCs in the selected warehouses.

\section{Table 9}

Pareto-optimal solutions

\begin{tabular}{ccccccc}
\hline No. & $\varepsilon_{1}$ & $\varepsilon_{3}$ & OF1 & OF2 & OF3 & Solution time (Sec) \\
\hline 1 & $3.71 \mathrm{E}+16$ & 0.787429 & $2.24 \mathrm{E}+17$ & 0.890433233 & 0.229070281 & 168.7005529 \\
2 & $7.43 \mathrm{E}+16$ & 0.984287 & $3.41 \mathrm{E}+17$ & 0.635568113 & 0.036337852 & 904.080983 \\
3 & $1.11 \mathrm{E}+17$ & 0.787429 & $2.16 \mathrm{E}+17$ & 0.881304983 & 0.229070281 & 269.9242233 \\
4 & $1.49 \mathrm{E}+17$ & 0.885858 & $2.99 \mathrm{E}+17$ & 0.579483842 & 0.132704066 & 155.3078006 \\
5 & $1.49 \mathrm{E}+17$ & 0.984287 & $2.99 \mathrm{E}+17$ & 0.524796561 & 0.036337852 & 329.8028742 \\
6 & $1.86 \mathrm{E}+17$ & 0.689001 & $2.05 \mathrm{E}+17$ & 0.895627968 & 0.325436496 & 2599.804964 \\
7 & $1.86 \mathrm{E}+17$ & 0.885858 & $2.61 \mathrm{E}+17$ & 0.570003038 & 0.132704066 & 162.4728741 \\
8 & $2.23 \mathrm{E}+17$ & 0.295286 & $2.01 \mathrm{E}+17$ & 0.889858993 & 0.710901355 & 1675.444653 \\
9 & $2.23 \mathrm{E}+17$ & 0.689001 & $2.11 \mathrm{E}+17$ & 0.897816086 & 0.325436496 & 296.0558163 \\
10 & $2.23 \mathrm{E}+17$ & 0.885858 & $2.24 \mathrm{E}+17$ & 0.558221378 & 0.132704066 & 248.3415905 \\
\hline
\end{tabular}




\begin{tabular}{ccccccc}
\hline No. & $\varepsilon_{1}$ & $\varepsilon_{3}$ & OF1 & OF2 & OF3 & Solution time (Sec) \\
\hline 11 & $2.6 \mathrm{E}+17$ & 0.984287 & $1.87 \mathrm{E}+17$ & 0.51015033 & 0.036337852 & 805.3396263 \\
12 & $2.97 \mathrm{E}+17$ & 0.787429 & $1.50 \mathrm{E}+17$ & 0.438424787 & 0.229070281 & 595.6295521 \\
13 & $3.34 \mathrm{E}+17$ & 0.984287 & $1.13 \mathrm{E}+17$ & 0.380959727 & 0.036337852 & 463.2417609 \\
\hline
\end{tabular}

\section{Table 10}

Parameters setting of PROMETHEE-II

\begin{tabular}{cccccc}
\hline \multirow{2}{*}{ Criteria } & & Criteria type & Preference/Weight & \multicolumn{2}{c}{ Thresholds } \\
\cline { 1 - 3 } \cline { 3 - 5 } OF1 & & Min & 0.3 & $1.14 \mathrm{E}+17$ & $2.28 \mathrm{E}+16$ \\
OF2 & & Max & 0.5 & 0.025842818 & 0.103371272 \\
OF3 & Min & 0.2 & 0.101184525 & 0.202369051 \\
\hline
\end{tabular}

\section{Table 11}

Obtained ranking of the Pareto-optimal solutions

\begin{tabular}{|c|c|c|c|c|c|}
\hline \multirow{2}{*}{ Pareto Solution } & \multicolumn{3}{|c|}{ Criteria } & \multirow{2}{*}{$\varphi(a)$} & \multirow{2}{*}{ Ranking } \\
\hline & OF1 & OF2 & OF3 & & \\
\hline 1 & $2.24 \mathrm{E}+17$ & 0.890433233 & 0.229070281 & 2.47619 & 2 \\
\hline 2 & $3.41 \mathrm{E}+17$ & 0.635568113 & 0.036337852 & -0.67377 & 7 \\
\hline 3 & $2.16 \mathrm{E}+17$ & 0.881304983 & 0.229070281 & 2.47619 & 3 \\
\hline 4 & $2.99 \mathrm{E}+17$ & 0.579483842 & 0.132704066 & -1.19996 & 9 \\
\hline 5 & $2.99 \mathrm{E}+17$ & 0.524796561 & 0.036337852 & -1.94435 & 11 \\
\hline 6 & $2.05 E+17$ & 0.895627968 & 0.325436496 & 2.057143 & 4 \\
\hline 7 & $2.61 \mathrm{E}+17$ & 0.570003038 & 0.132704066 & -1.08339 & 8 \\
\hline 8 & $2.01 \mathrm{E}+17$ & 0.889858993 & 0.710901355 & 1.40000 & 6 \\
\hline 9 & $2.11 \mathrm{E}+17$ & 0.897816086 & 0.325436496 & 2.057143 & 5 \\
\hline 10 & $2.24 E+17$ & 0.558221378 & 0.132704066 & 4.181088 & 1 \\
\hline 11 & $1.87 \mathrm{E}+17$ & 0.51015033 & 0.036337852 & -1.32772 & 10 \\
\hline 12 & $1.50 \mathrm{E}+17$ & 0.438424787 & 0.229070281 & -5.57281 & 13 \\
\hline 13 & $1.13 E+17$ & 0.380959727 & 0.036337852 & -2.84576 & 12 \\
\hline
\end{tabular}

Table 12

The opened warehouses and the corresponding amount of the RCs

\begin{tabular}{|c|c|c|c|c|c|c|}
\hline \multirow{2}{*}{$\mathrm{RC}$} & \multicolumn{6}{|c|}{ Warehouse } \\
\hline & 1 & 2 & 3 & 4 & 5 & 6 \\
\hline 1 & $2.1000 \mathrm{E}+8$ & $1.1527 \mathrm{E}+8$ & $1.7618 \mathrm{E}+8$ & $2.1000 \mathrm{E}+8$ & $2.1000 \mathrm{E}+8$ & $1.3564 \mathrm{E}+8$ \\
\hline 2 & $2.1000 \mathrm{E}+8$ & $1.8590 \mathrm{E}+8$ & $1.0683 \mathrm{E}+8$ & $2.1000 \mathrm{E}+8$ & $2.1000 \mathrm{E}+8$ & $8.3325 \mathrm{E}+7$ \\
\hline 3 & $2.1000 \mathrm{E}+9$ & $2.1000 \mathrm{E}+9$ & $1.5306 \mathrm{E}+9$ & $2.1000 \mathrm{E}+9$ & $2.1000 \mathrm{E}+9$ & $1.1846 \mathrm{E}+9$ \\
\hline 4 & $4.2000 \mathrm{E}+8$ & $4.2000 \mathrm{E}+8$ & $1.0103 \mathrm{E}+8$ & $4.2000 \mathrm{E}+8$ & $4.2000 \mathrm{E}+8$ & $1.7334 \mathrm{E}+8$ \\
\hline 5 & $2.1000 \mathrm{E}+8$ & $2.1000 \mathrm{E}+8$ & $1.4706 \mathrm{E}+8$ & $2.1000 \mathrm{E}+8$ & $2.1000 \mathrm{E}+8$ & $5.9524 \mathrm{E}+7$ \\
\hline
\end{tabular}

To show the differences of the obtained Pareto-optimal solutions, we investigate the situations of DP \#14 (as the high-prioritized DP) and DP \#22 (as the less-prioritized DP), as two instances, in the first and second ranked Pareto-optimal solutions. Fig. 14 depicts the situations of these DPs in Scenario \#8 with regard to RC 3 (i.e., medical kit). It should be considered that the priorities of RC 3 in Scenario \#8 for DP \#14 and DP \#22 are 0.0622 $\times 0.5$ and $0.0167 \times 0.5$, respectively. As can be seen in Fig. 14, when the efficacy is 
increased (i.e., OF2), the MR utility level at the high-prioritized DP (i.e., DP \#14) is improved while the MR utility level at the less-prioritized DP (i.e., DP \#22) is got worse. Moreover, the imbalance measure is got worse from the first ranked Pareto solution to the second one. Therefore, if the DM seeks to balance, he/she should select the first ranked solution, but if he/she prefers efficacy, the second ranked Pareto solution is better than the first one. Similar insights can be provided by comparing the different Paretooptimal solutions, but the DM should pick one of them regarding to his/her preferences to the different objectives.

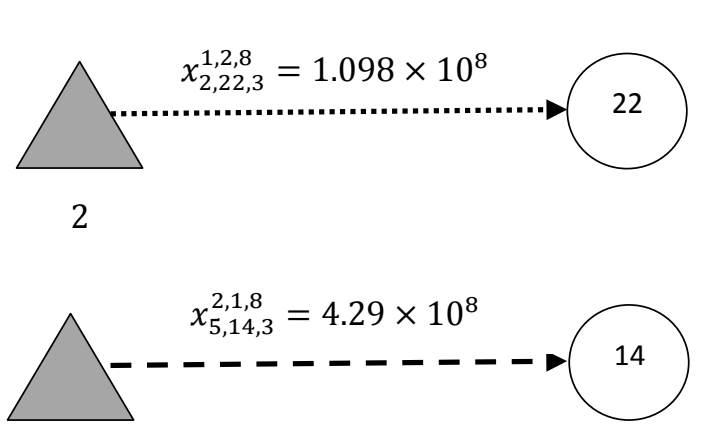

5

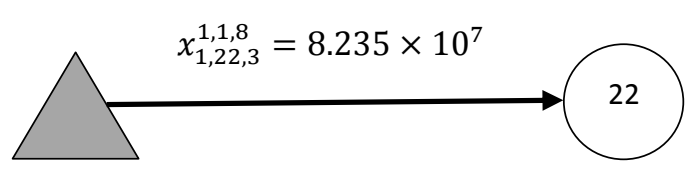

1

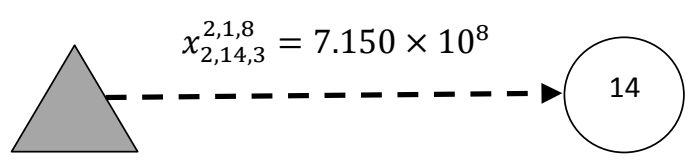

2

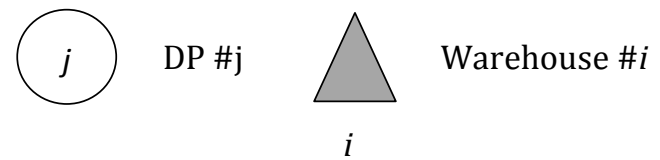

$$
\begin{aligned}
& d_{22,3,8}=1.098 \times 10^{8} \\
& \tau_{22,8}=0.0167 \\
& \xi_{3}=0.5 \\
& \varphi_{22,8}=0.5 \\
& d_{14,3,8}=7.150 \times 10^{8} \\
& \tau_{14,8}=0.0622 \\
& \xi_{3}=0.5 \\
& \varphi_{14,8}=0.0108
\end{aligned}
$$

$$
\begin{aligned}
& d_{22,3,8}=1.098 \times 10^{8} \\
& \tau_{22,8}=0.0167 \\
& \xi_{3}=0.5 \\
& \varphi_{22,8}=0.0014
\end{aligned}
$$

$d_{14,3,8}=7.150 \times 10^{8}$

$\tau_{14,8}=0.0622$

$\xi_{3}=0.5$

$\varphi_{14,8}=0.0286$

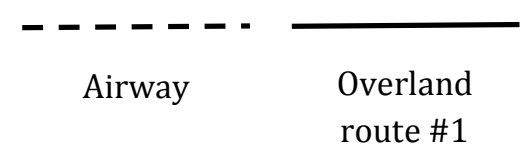

Overland route \#2

Fig. 14. Situation of DP \#14 vs. DP \#22 with regard to the first and second ranked Pareto solutions

To analyze the sensitivity of PROMETHEE-II, we investigate 36 different combinations of weights (weights are in range [0.1-0.8]; step by increments of 0.1 subject to $\sum_{i=1}^{3} w_{i}=1$ ). Noteworthy, the first ranked solution (i.e., Solution \#10) is fixed in the most of the different combinations (see Table 13). Solution \#13 is placed as the first ranked when the importance weight of OF2 changes to 0.1. Solution \#13 also selects all the warehouses, but it stocks the RCs around 19\% less than Solution \#10. Accordingly, the efficacy of Solution \#13 is lower than Solution \#10 around 32\%. It should be noted that setting such 
a low weight, 0.1 , to the most important objective (regarding the DM's point of view) is such a strategic mistake, even in the situation of a huge cost saving $49.5 \%$. Moreover, for two combinations of weights $\left(w_{1}=0.1, w_{2}=0.2, w_{3}=0.7\right)$ and $\left(w_{1}=0.1, w_{2}=0.1, w_{3}=0.8\right)$, the first ranked solution is Solution \#2 and Solution \#11, respectively. Solution \#11 can be chosen by someone that prefers efficiency and balance to efficacy. Because the total cost of this solution is almost $16.52 \%$ lower than Solution \#10, but its balance is better than Solution \#10 around 72\% while the both solutions almost have same amount of efficacy. The total cost of Solution \#2 is higher than Solution \#10 around 34.31\%; however, this solution presents a higher amount of efficacy (i.e., 12.17\%) and balance (i.e., 72.62\%). Therefore, this solution will be a great one if someone does not care of the network cost.

In conclusion, Solution \#10 is a reliable decision with regard to different preferences of the DM unless when the lowest preference (i.e., 0.1) is assigned to OF2 (i.e., efficacy), while that is the most important objective function.

Table 13. Ranking of the Pareto optimal solutions by considering different weights for the objectives

\begin{tabular}{|c|c|c|c|c|c|c|c|c|c|c|c|c|c|c|c|}
\hline \multicolumn{3}{|c|}{ Weight } & \multicolumn{13}{|c|}{ Rank } \\
\hline$w_{1}$ & $w_{2}$ & $w_{3}$ & 1 & 2 & 3 & 4 & 5 & 6 & 7 & 8 & 9 & 10 & 11 & 12 & 13 \\
\hline 0.8 & 0.1 & 0.1 & S13 & S10 & S11 & $\mathrm{S} 1$ & S3 & S12 & S6 & S9 & S8 & S7 & S5 & S4 & S2 \\
\hline 0.7 & 0.2 & 0.1 & S10 & $\mathrm{S} 1$ & S3 & $\mathrm{S} 13$ & S6 & S9 & S8 & $\mathrm{S} 11$ & $\mathrm{~S} 12$ & S7 & S4 & S5 & S2 \\
\hline 0.7 & 0.1 & 0.2 & $\mathrm{~S} 13$ & S10 & S11 & $\mathrm{S} 1$ & S3 & S7 & $\mathrm{S} 12$ & S5 & S6 & S9 & S4 & S8 & S2 \\
\hline 0.6 & 0.2 & 0.2 & $\mathrm{~S} 10$ & S13 & S11 & $\mathrm{S} 1$ & S3 & S6 & S9 & S7 & S8 & S5 & S4 & S12 & S2 \\
\hline 0.6 & 0.3 & 0.1 & $\mathrm{~S} 10$ & $\mathrm{~S} 1$ & S3 & S6 & S9 & S8 & $\mathrm{S} 13$ & S11 & S7 & S4 & $\mathrm{S} 12$ & S5 & S2 \\
\hline 0.6 & 0.1 & 0.3 & S13 & S10 & S11 & S5 & S7 & S4 & S1 & S3 & $\mathrm{S} 12$ & S6 & S9 & S8 & S2 \\
\hline 0.5 & 0.4 & 0.1 & $\mathrm{~S} 10$ & $\mathrm{~S} 1$ & S3 & S6 & S9 & S8 & S11 & S7 & $\mathrm{S} 13$ & S4 & S5 & $\mathrm{S} 12$ & S2 \\
\hline 0.5 & 0.3 & 0.2 & $\mathrm{~S} 10$ & S1 & S3 & S6 & S9 & $\mathrm{S} 13$ & S11 & S8 & S7 & S4 & S5 & S2 & $\mathrm{S} 12$ \\
\hline 0.5 & 0.2 & 0.3 & S10 & S13 & S11 & S5 & S7 & S1 & S3 & S4 & S6 & S9 & S8 & S2 & S12 \\
\hline 0.5 & 0.1 & 0.4 & S13 & S11 & S10 & S5 & S7 & S4 & S2 & S1 & S36 & $\mathrm{S} 12$ & S6 & S9 & S8 \\
\hline 0.4 & 0.5 & 0.1 & S10 & $\mathrm{S} 1$ & S3 & S6 & S9 & S8 & S7 & S4 & S11 & S2 & S5 & S13 & $\mathrm{S} 12$ \\
\hline 0.4 & 0.4 & 0.2 & S10 & $\mathrm{S} 1$ & S3 & S6 & S9 & S8 & S11 & S7 & S4 & S13 & S5 & S2 & $\mathrm{S} 12$ \\
\hline 0.4 & 0.3 & 0.3 & $\mathrm{~S} 10$ & S11 & S13 & $\mathrm{S} 1$ & S3 & S7 & S5 & S6 & S9 & S4 & S2 & S8 & $\mathrm{S} 12$ \\
\hline 0.4 & 0.2 & 0.4 & $\mathrm{~S} 10$ & S13 & S11 & S5 & S7 & S4 & S2 & S1 & S3 & S6 & S9 & S8 & $\mathrm{S} 12$ \\
\hline 0.4 & 0.1 & 0.5 & $\mathrm{~S} 13$ & S11 & S10 & S5 & S7 & S4 & S2 & S1 & S3 & $\mathrm{S} 12$ & S6 & S9 & S8 \\
\hline 0.3 & 0.6 & 0.1 & $\mathrm{~S} 10$ & $\mathrm{~S} 1$ & S3 & S6 & S9 & S8 & S2 & S7 & S4 & S11 & S5 & S13 & $\mathrm{S} 12$ \\
\hline 0.3 & 0.5 & 0.2 & $\mathrm{~S} 10$ & $\mathrm{~S} 1$ & S3 & S6 & S9 & S8 & S2 & S7 & S4 & S11 & S5 & S13 & $\mathrm{S} 12$ \\
\hline 0.3 & 0.4 & 0.3 & S10 & $\mathrm{S} 1$ & S3 & S6 & S9 & S11 & S2 & S7 & S4 & S5 & S8 & S13 & S12 \\
\hline 0.3 & 0.3 & 0.4 & S10 & S11 & S13 & S5 & S2 & S7 & S4 & S1 & S3 & S6 & S9 & S8 & S12 \\
\hline 0.3 & 0.2 & 0.5 & S10 & S11 & S13 & S5 & S2 & S7 & S4 & S1 & S3 & S6 & S9 & S8 & S12 \\
\hline 0.3 & 0.1 & 0.6 & S13 & S11 & S5 & S10 & S2 & S7 & S4 & S1 & S3 & $\mathrm{S} 12$ & S6 & S9 & S8 \\
\hline 0.2 & 0.7 & 0.1 & S10 & $\mathrm{S} 1$ & S3 & S6 & S9 & S8 & S2 & S4 & S7 & S11 & S5 & S13 & S12 \\
\hline 0.2 & 0.6 & 0.2 & $\mathrm{~S} 10$ & $\mathrm{~S} 1$ & S3 & S6 & S9 & S8 & S2 & S4 & S7 & S11 & S5 & S13 & S12 \\
\hline 0.2 & 0.5 & 0.3 & $\mathrm{~S} 10$ & $\mathrm{~S} 1$ & S3 & S2 & S6 & S9 & S8 & S7 & S4 & S11 & S5 & S13 & S12 \\
\hline 0.2 & 0.4 & 0.4 & $\mathrm{~S} 10$ & S2 & S11 & S5 & $\mathrm{S} 1$ & S3 & S7 & S4 & $\mathrm{S} 13$ & S6 & S9 & S8 & $\mathrm{S} 12$ \\
\hline 0.2 & 0.3 & 0.5 & $\mathrm{~S} 10$ & S2 & S11 & S5 & S13 & S7 & S4 & S1 & S3 & S6 & S9 & S8 & $\mathrm{S} 12$ \\
\hline 0.2 & 0.2 & 0.6 & S10 & S11 & S13 & S5 & S2 & S7 & S4 & S1 & S3 & S6 & S9 & S12 & S8 \\
\hline 0.2 & 0.1 & 0.7 & S13 & S11 & S5 & S2 & S10 & S7 & S4 & S1 & S3 & S12 & S6 & S9 & S8 \\
\hline 0.1 & 0.8 & 0.1 & S10 & $\mathrm{S} 1$ & S3 & S6 & S9 & S8 & S2 & S4 & S7 & S5 & S11 & S13 & S12 \\
\hline 0.1 & 0.7 & 0.2 & S10 & $\mathrm{S} 1$ & S3 & S6 & S9 & S8 & S2 & S4 & S7 & S5 & S11 & S13 & S12 \\
\hline 0.1 & 0.6 & 0.3 & S10 & $\mathrm{S} 1$ & S3 & S2 & S6 & S9 & S8 & S4 & S7 & S5 & S11 & S13 & S12 \\
\hline 0.1 & 0.5 & 0.4 & S10 & S2 & S1 & S3 & S11 & S5 & S4 & S6 & S9 & S7 & S8 & S13 & S12 \\
\hline 0.1 & 0.4 & 0.5 & S10 & S2 & S11 & S5 & S4 & S7 & $\mathrm{S} 13$ & S1 & S3 & S6 & S9 & S8 & $\mathrm{S} 12$ \\
\hline 0.1 & 0.3 & 0.6 & $\mathrm{~S} 10$ & S2 & S11 & S5 & S13 & S4 & S7 & S1 & S3 & S6 & S9 & S8 & S12 \\
\hline 0.1 & 0.2 & 0.7 & S2 & S11 & S5 & S10 & S13 & S7 & S4 & S1 & S3 & S6 & S9 & $\mathrm{S} 12$ & S8 \\
\hline
\end{tabular}




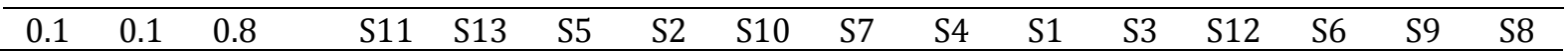

To show the impact of the usage possibility of a set of alternative routes for each of the applied transportation modes (i.e. multi-mode-route feature) on the efficiency, efficacy, and imbalance measures, three states are defined for the case study as follows:

State (i): Multi-mode: There are two vehicle types, i.e. truck and helicopter, to be applied in the disaster area. Each of them can just use one specific route between each warehouse-DP pair.

State (ii): Multi-route: There are two overland alternative routes that can be passed by the trucks between each warehouse-DP pair.

State (iii): Multi-mode-route: Integration of States (i) and (ii).

Fig. 15 illustrates the differences of the considered measures among the tree defined states. As can be seen, State (iii) achieved the best values for all the measures. Indeed, employing a transportation system with the multi-route-mode feature improved the efficiency, efficacy, and imbalance measures around 51\%, 55\%, and 89\% on average, respectively. As can be derived, we could design a more effective and balanced network while spending a lower amount of money. The efficacy and imbalance measures were improved because of increasing the transportation capacity. Noteworthy, although using the helicopters had more cost than trucks per unit of time, the total cost decreased because of a huge improvement in the average of delivery time.

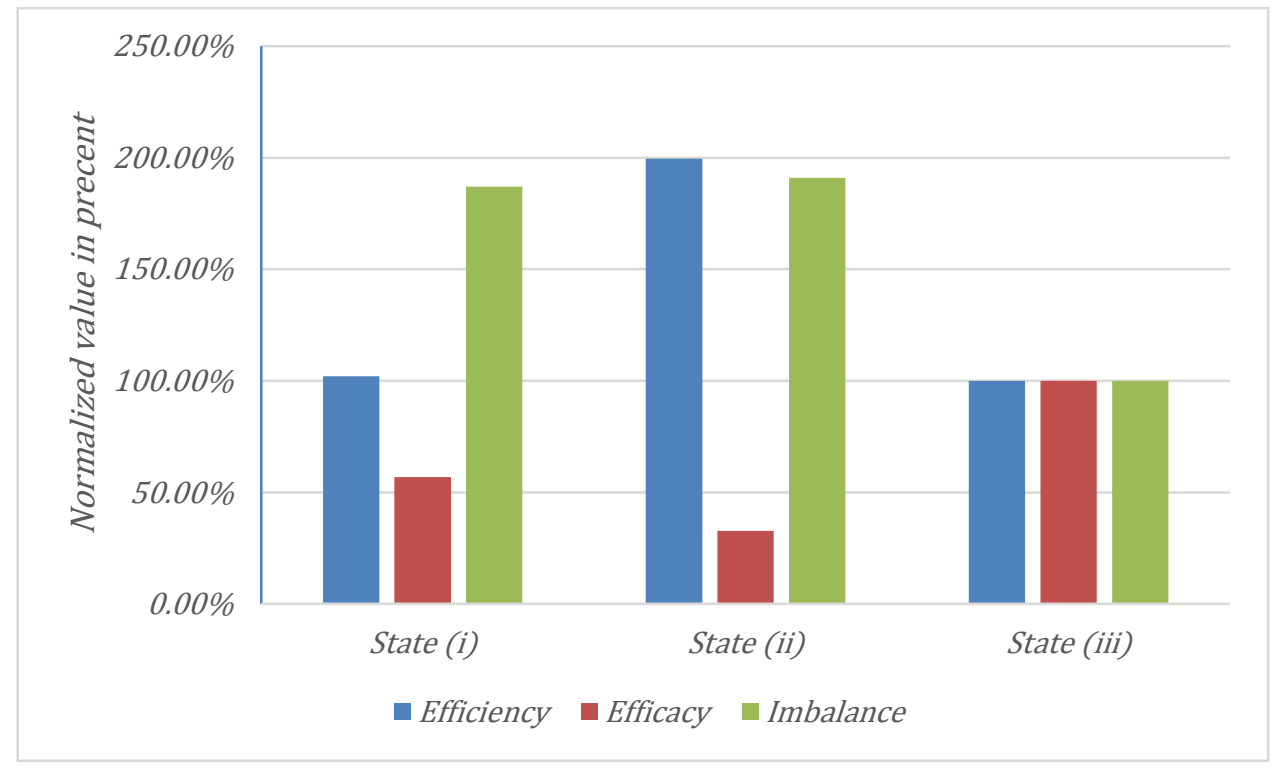

Fig. 15. Differences of the measures regarding the different employed states

\section{Conclusion and future research direction}

This work has proposed a multi-objective, two-stage stochastic, non-linear, and mixedinteger mathematical model to design a humanitarian relief logistics network considering the three objectives, i.e. efficiency, efficacy, and imbalance, to be optimized.

A new utility level of delivered relief commodities, called MR utility level, has been developed to measure the benefit level of each demand point at disaster area. The MR utility level simultaneously takes account the importance of the delivery time, the 
priorities of the different relief commodities and demand point, and the fraction of the satisfied demand at each specific demand point. Improved versions of the efficacy and imbalance measures have been presented based on the MR utility level. An integrated feature of the usage possibility of alternative routes for each applied transportation mode (i.e. the multi-mode-route feature) has been incorporated into the presented mathematical model to increase the responsiveness and reliability of the humanitarian relief logistics network. The developed multi-objective and non-linear mathematical model has been solved by employing an integrated separable programming-augmented $\varepsilon$-constraint (ISPAE) approach.

The impacts of the MR utility level and the efficacy and imbalance measures on the network design, have been investigated through a random experiment. In this regard, four new indices have been defined and their improvements through applying the new measures and utility level has been proved. In addition, to be more confident to the ISPAE approach, this method and the NSGA-II algorithm have been compared through some random experiments. The results showed that the ISPAE method has a better timeperformance than NSGA-II, while producing solutions of similar high quality. Furthermore, NSGA-II is incapable to solve the large-sized problems.

To show the applicability of the developed model and solution approach, a real case study on earthquake preparedness for Tehran, has been investigated. The achieved improvement by employing the new utility level and measures has been justified through analyzing the set of Pareto-optimal solutions. Moreover, our investigations showed that if the network uses the multi-mode-route feature, it can improve the efficiency, efficacy, and imbalance measure around 51\%, 55\%, and $89 \%$ on average, respectively. In other words, a more effective and balanced network is designed with a lower amount of money.

Although this work approaches the real disaster situations and problems, there are many practical issues that should be handled in future research directions. For instance, taking into account the availability of the needed personnel for relief operations (e.g., the shipment of relief commodities, and treating injured individuals in the hospitals) in the network when a disaster occurs, could be a way to improve the realistic features of the humanitarian relief logistics problem.

\section{References}

Ahmadi, M., Seifi, A., Tootooni, B., 2015. A humanitarian logistics model for disaster relief operation considering network failure and standard relief time: A case study on San Francisco district. Transportation Research Part E: Logistics and Transportation Review, 75, 145-163.

Akkihal, A.R., 2004. Inventory pre-positioning for humanitarian operations. MS thesis, Massachusetts Institute of Technology.

Altay, N., Green III, W.G., 2006. OR/MS research in disaster operations management. European Journal of Operational Research, 175(1), 475-93.

Azadeh, A., Rezaei-Malek, M., Evazabadian, F., Sheikhalishahi M., 2015. Improved design of CMS by considering operators decision-making styles. International Journal of Production Research, 53(11), 3276-3287. 
Balcik, B., Beamon, B.M., 2008. Facility location in humanitarian relief. International Journal of Logistics Research and Applications, 11(2), 101-121.

Barzinpour, F., Esmaeili, V., 2014. A multi-objective relief chain location distribution model for urban disaster management. International Journal of Advanced Manufacturing Technology, 70(5-8), 1291-1302.

Bozorgi-Amiri, A., Jabalameli, M.S., Alinaghian, M., Heydari, M., 2012. A modified particle swarm optimization for disaster relief logistics under uncertain environment. International Journal of Advanced Manufacturing Technology, 60, 357-371.

Bozorgi-Amiri, A., Jabalameli, M., Mirzapour Al-e-Hashem, S., 2013. A multi-objective robust stochastic programming model for disaster relief logistics under uncertainty. OR Spectrum, 35(4), 905-933.

Bozorgi-Amiri, A., Asvadi, S., 2015. A prioritization model for locating relief logistic centers using analytic hierarchy process with interval comparison matrix. Knowledge-Based Systems, 86, 173-181.

Caunhye, A.M., Nie, X., Pokharel, S., 2012. Optimization models in emergency logistics: A literature review. Socio-Economic Planning Sciences, 46(1), 4-13.

Chang, M.S., Tseng, Y.L., Chen, J.W., 2007. A scenario planning approach for the flood emergency logistics preparation problem under uncertainty. Transportation Research Part E: Logistics and Transportation Review, 43(6), 737-54.

Davis, L.B., Samanlioglu, F., Qu, X., Root, S., 2013. Inventory planning and coordination in disaster relief efforts. International Journal of Production Economics, 141, 561-573.

Döyen, A., Aras, N., Barbarosoglu, G., 2012. A two-echelon stochastic facility location model for humanitarian relief logistics. Optimization Letters, 6(6), 1123-1145.

Duran, S., Gutierrez, M.A., Keskinocak, P., 2011. Pre-positioning of emergency items for CARE international. Interfaces, 41(3), 223-237.

Edrissi, A., Poorzahedy, H., Nassiri, H., Nourinejad, M., 2013. A multi-agent optimization formulation of earthquake disaster prevention and management. European Journal of Operational Research, 229, 261-275.

Falasca, M., Zobel, C.W., 2011. A two-stage procurement model for humanitarian relief supply chains. Journal of Humanitarian Logistics and Supply Chain Management, 1 (2), 151-169.

Galindo, G., Batta, R., 2013. Review of recent developments in OR/MS research in disaster operations management. European Journal of Operational Research, 230(2), 201211.

Garrido, R.A., Lamas, P., Pino, F.J., 2014. A stochastic programming approach for floods emergency logistics. Transportation Research Part E: Logistics and Transportation Review, 75, 18-31.

Gralla, E., Goentzel, J., Fine, C., 2014. Assessing trade-offs among multiple objectives for humanitarian aid delivery using expert preferences. Production and Operations Management, 23, 978-989.

Guha-Sapir, D., Hoyois, P., Below, R., 2014. Annual disaster statistical review 2013: the numbers and trends. Available at: http://cred.be/sites/default/files/ADSR 2013.pdf (last accessed May 12, 2015). 
Gutjahr, W.J., Nolz, P.C., 2016. Multicriteria optimization in humanitarian aid. European Journal of Operational Research, 252(2), 351-366.

Hamedi, M., Haghani, A., Yang, S., 2012. Reliable transportation of humanitarian supplies in disaster response: Model and heuristic. Procedia - Social and Behavioral Sciences, $54,1205-1219$.

Holguín-Veras, J., Pérez, N., Jaller, M., Van Wassenhove, L. N., Aros-Vera, F., 2013. On the appropriate objective function for post-disaster humanitarian logistics models. Journal of Operations Management, 31(5), 262-280.

Hoyos, M.C., Morales, R.S., Akhavan-Tabatabaei, R., 2015. OR models with stochastic components in disaster operations management: A literature survey. Computers and Industrial Engineering, 82, 183-197.

Huang, Y., Fan, Y., 2011. Modeling uncertainties in emergency service resource allocation. Journal of Infrastructure Systems, 17(1), 35-41.

Huang, M., Smilowitz, K., Balcik, B., 2012. Models for relief routing: Equity, efficiency and efficacy. Transportation Research Part E: Logistics and Transportation Review, 48(1), 2-18.

Iakovou, E., Ip, C.M., Douligeris, C., Korde, A., 1997. Optimal location and capacity of emergency cleanup equipment for oil spill response. European Journal of Operational Research, 96(1), 72-80.

Japan International Cooperation Agency (JICA), (2010). The Study on Seismic Microzoning of the Greater Tehran Area in the Islamic Republic of Iran, Centre for Earthquake and Environmental Studies of Tehran and Tehran-Municipality, Tehran, November.

Jia, H., Ordonez, F., Dessouky, M.M., 2007. Solution approaches for facility location of medical supplies for large-scale emergencies. Computers and Industrial Engineering, 52(2), 257-276.

Karsu, O., Morton, A., 2015. Inequity averse optimisation in operational research. European Journal of Operational Research, 245(2), 343-359.

Lee, W.S., Kim, B.S., Opit, P.F., 2014. A stock pre-positioning model to maximize the total expected relief demand of disaster areas. Industrial Engineering and Management Systems, 13(3), 297-303.

Li, A.C.Y., Xu, N., Nozick, L., Davidson, R., 2011. Bilevel optimization for integrated shelter location analysis and transportation planning for hurricane events. Journal of Infrastructure Systems, 17(4), 184-192.

Mavrotas, G., 2009. Effective implementation of the $\varepsilon$-constraint method in Multi Objective Mathematical Programming problems. Applied Mathematics and Computation, 213(2), 455-465.

McCall, V.M., 2006. Designing and prepositioning humanitarian assistance pack-up kits (HA PUKs) to support pacific fleet emergency relief operations. MS Thesis, Naval Postgraduate School, Monterey, California.

Mete, H.O., Zabinsky, Z.B., 2010. Stochastic optimization of medical supply location and distribution in disaster management. International Journal of Production Economics, 126(1), 76-84. 
Nagurney, A., Masoumi, A.H., Yu, M., 2015. An integrated disaster relief supply chain network model with time targets and demand uncertainty. In Regional Science Matters: Studies Dedicated to Walter Isard, P. Nijkamp, A. Rose, and K. Kourtit, Editors, Springer International Publishing Switzerland, 287-318.

Nateghi, F., 2001. Earthquake scenario for the megacity of Tehran. Disaster Prevention and Management, 10(2), 95-101.

Özdamar, L., Ertem, M.A., 2015. Models, solutions and enabling technologies in humanitarian logistics. European Journal of Operational Research, 244(1), 55-65.

Psaraftis, H.N., Tharakan, G.G., Ceder, A., 1986. Optimal response to oil spills: the strategic decision case. Operations Research, 34(2), 203-217.

Rawls, C.G., Turnquist, M.A., 2010. Pre-positioning of emergency supplies for disaster response. Transportation Research Part B: Methodological, 44(4), 521-534.

Rawls, C.G., Turnquist, M.A., 2011. Pre-positioning planning for emergency response with service quality constraints. OR Spectrum, 33(3), 481-498.

Rawls, C.G., Turnquist, M.A., 2012. Pre-positioning and dynamic delivery planning for short-term response following a natural disaster. Socio-Economic Planning Sciences, 46(1), 46-54.

Rezaei-Malek, M., Tavakkoli-Moghaddam, R., 2012. A new multi-objective mathematical model for relief logistic network under uncertainty. Proceeding of the $6^{\text {th }}$ IEEE International Conference on Industrial Engineering and Engineering Management (IEEE-IEEM2012), Hong Kong, December 12, 1878-1882.

Rezaei-Malek, M., Tavakkoli-Moghaddam, R., 2014. Robust humanitarian relief logistics network planning. Uncertain Supply Chain Management, 2(2), 73-96.

Rezaei-Malek, M., Tavakkoli-Moghaddam, R., Salehi, N., 2014. Robust planning of medical supplies with time windows in a humanitarian relief logistic network. Proceeding of the $4^{\text {th }}$ International Conference on Computers and Industrial Engineering (CIE44). Istanbul, Turkey, October 14-16, 134-147.

Rezaei-Malek, M., Tavakkoli-Moghaddam, R., Zahiri, B., Amiri-Bozorgi, A., 2016. An interactive approach for designing a robust disaster relief logistics network with perishable commodities. Computers and Industrial Engineering, 94, 201-215.

Rezaei-Malek, M., Razmi, J., Tavakkoli-Moghaddam, R., Taheri-Moghaddam, A., 2016. Towards a psychologically-consistent cellular manufacturing system. International Journal of Production Research, forthcoming.

Rodriguez-Espindola, O., Gaytan, J., 2015. Scenario-based preparedness plan for floods. Natural Hazards, 76(2), 1241-1262.

Ruan, J., Wang, X., Shi, Y. (2014). A two-stage approach for medical supplies intermodal transportation in large-scale disaster responses. International Journal of Environmental Research and Public Health, 11, 11081-11109.

Salehi Sadghiani, N., Torabi, S.A., Sahebjamnia, N., 2015. Retail supply chain network design under operational and disruption risks. Transportation Research Part E: Logistics and Transportation Review, 75, 95-114.

Sedighy, S.Q., Shakouri G., H., Rezaei-Malek, M., 2015. A novel multi-objective mathematical model for planning an auxiliary public transportation network: A 
covering-routing approach. Economic Computation and Economic Cybernetics Studies and Research, 49(1), 239-262.

Sheu, J-B., 2010. Dynamic relief-demand management for emergency logistics operations under large-scale disasters. Transportation Research Part E: Logistics and Transportation Review, 46(1), 1-17.

Thomas, A., Mizushima, M., 2005. Logistics training: necessity or luxury?, Forced Migration Review, 22, 60-61.

Tofighi, S., 2011. A logistics planning model and solution method in humanitarian relief chains. M.Sc. Thesis, School of Industrial Engineering, University of Tehran.

Tofighi, S., Torabi, S.A., Mansouri, S.A., 2011. A fuzzy stochastic approach for prepositioning and distribution of emergency supplies in disaster management. Proceeding of the $22^{\text {nd }}$ POMS Annual Conference, Reno, Nevada, U.S.A., April 29 to May 2.

Tofighi, S., Torabi, S.A., Mansouri, S.A., 2015. Humanitarian logistics network design under mixed uncertainty. European Journal of Operational Research, 250, 239-250.

Torabi, S.A., Baghersad, M., Mansouri, S.A., 2015. Resilient supplier selection and order allocation under operational and disruption risks. Transportation Research Part E: Logistics and Transportation Review, 79, 22-48.

Turcksina, L., Bernardinia, A., Macharisa, C., 2011. A combined AHP-PROMETHEE approach for selecting the most appropriate policy scenario to stimulate a clean vehicle fleet. Procedia Social and Behavioral Sciences, 20, 954-965.

Tzeng, G., Cheng, H., Huang, T., 2007. Multi-objective optimal planning for designing relief delivery systems. Transportation Research Part E: Logistics and Transportation Review, 6, 673-686.

Tzeng, G-H., Huang, J-J., 2011. Multiple attribute decision making: Methods and applications. CRC Press, Taylor \& Francis Group.

Vitoriano, B., Ortuño, M.T., Tirado, G., Montero, J., 2011. A multi-criteria optimization model for humanitarian aid distribution. Journal of Global Optimization, 51(2), 189208.

Wilhelm, W.E., Srinivasa, A.V., 1996. A strategic, area-wide contingency planning model for oil spill cleanup operations with application demonstrated to the Galveston Bay area. Decision Sciences, 27(4), 767-799.

Williams, H. P., 2013. Model building in mathematical programming. John Wiley \& Sons Ltd, West Sussex, England.

Zhalechian, M., Tavakkoli-Moghaddam, R., Zahiri, B., Mohammadi, M., 2016. Sustainable design of a closed-loop location-routing-inventory supply chain network under mixed uncertainty. Transportation Research Part E: Logistics and Transportation Review, 89, 182-214. 